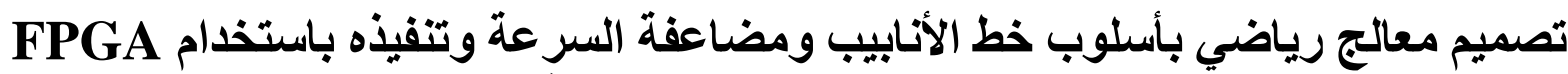

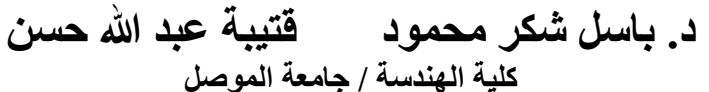

$$
\begin{aligned}
& \text { الخلاصة }
\end{aligned}
$$

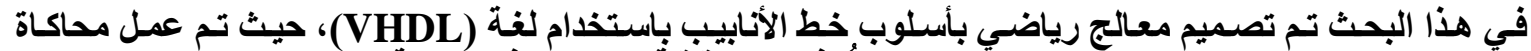

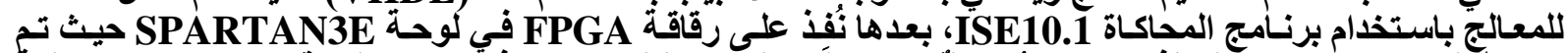

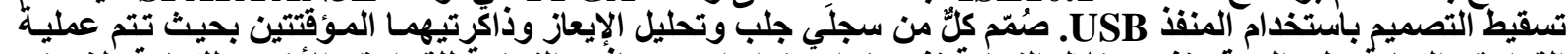

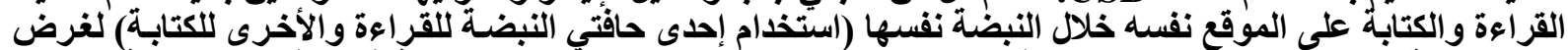

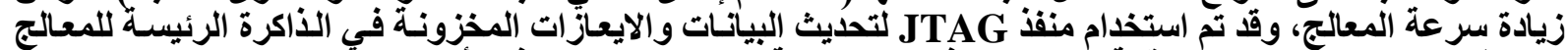

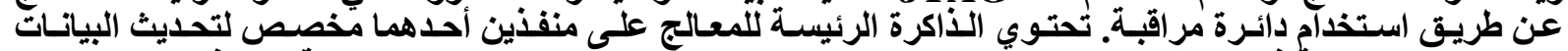

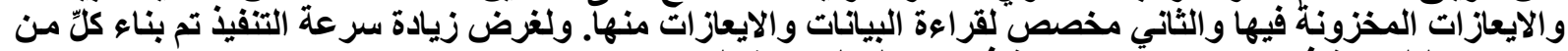

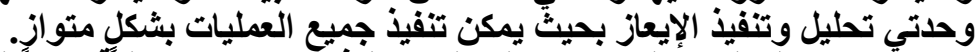

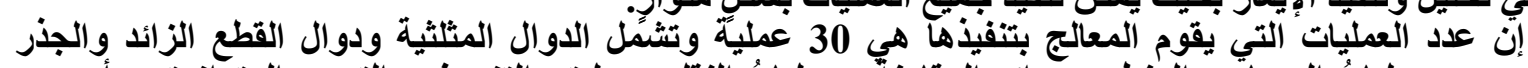

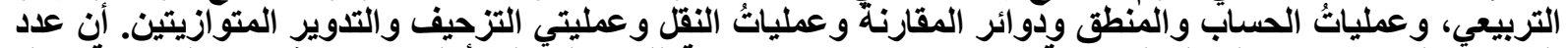

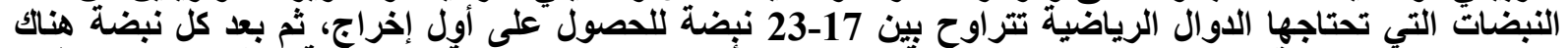

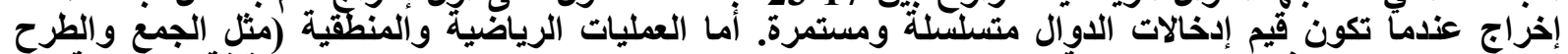

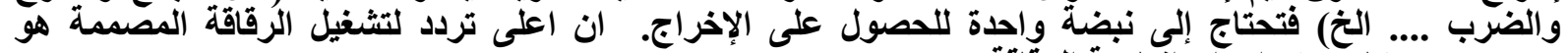

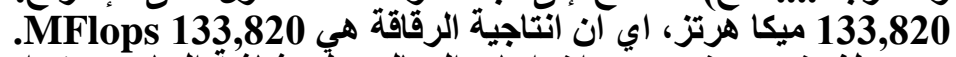

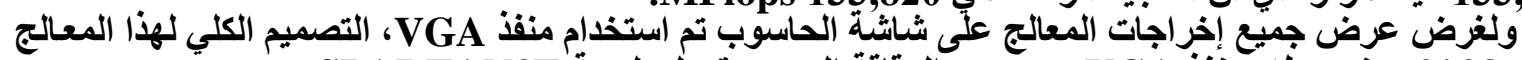

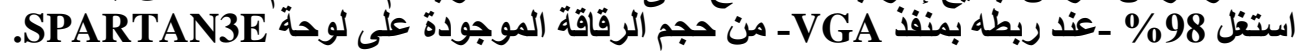

\title{
Design a piplined math processor, doubling its speed and implementing it on FPGA
}

\author{
Basil Shukr Mahmood(Ph.D.) \\ Prof. of microprocessors \\ University of Mosul / Mosul / Iraq \\ Basil_mahmood@yahoo.com \\ Qutaiba Abdullah Hassan \\ Msc. of Computer Eng. \\ Quteiba_qa@yahoo.com
}

\section{Abstract}

In this paper, a pipeline math processor is designed using VHDL, where doing simulation for processor by using simulation program ISE10.1. The processor is implemented on the FPGA chip in a panel SPARTAN3E, where it has been downloaded by using USB port. The register and buffer memory of each of the fetch and decoder units are designed such that reading and writing operations for the same location are performed during one clock cycle (each clock pulse edge is used for one operation). JTAG port is used to update the data and instructions stored in the main memory via monitor circuit. The main memory of the processor contains two ports, one of them is used to update the data and instructions and the other is used to read data and instructions. For the purpose of increasing the speed, decode and execute units are built so that all operations can be executed in parallel.

The number of operations that can be executed on the processor are 30 operations including triangular functions, hyperbolic functions, square root, ALU, comparison operations, move operation, and parallel shifting and rotation operations. The number of clocks that is required by the mathematical functions, ranges between 17-23 clocks for the first output and then each clock has it's output when the values of input functions are sequential and continuous. The remaining operations (such as addition, subtraction, multiplication and division) need only one clock for each output. The maximum operating frequency for the design chip was found to be $133.820 \mathrm{MHz}$, therefore its throughput is 133.820 MFlops. For the purpose of displaying all the processor outputs on a computer screen, the VGA Port is used. The overall design of this processor occupies $98 \%$ ( when the processor is connected with VGA port) from the volume of the FPGA chip on board SPARTAN3E. 
في هذا البحث تم تصميم معالج رياضي باسلوب خط الانابيب وتنفيذه باستخدام رقاقة FPGA كما تم استخدام الحافة

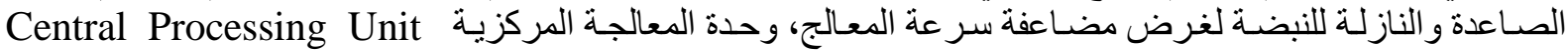

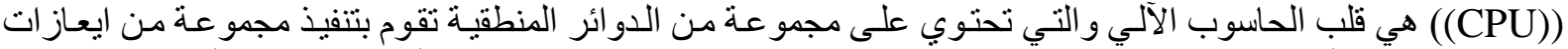

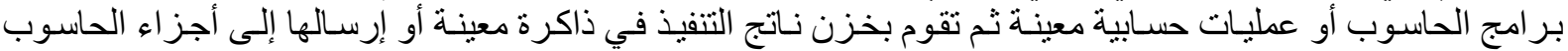

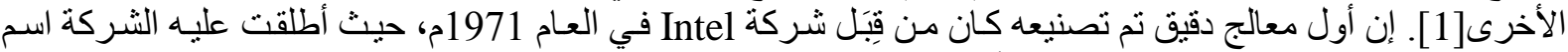

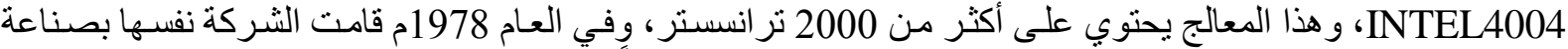

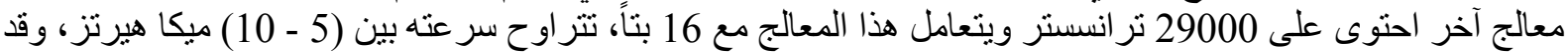
أطلقت الشركة عليه اسم آلى INTEL8088 و الذي استخدمته شركة IBM لتصنيع أول حاسوب آلي [2].

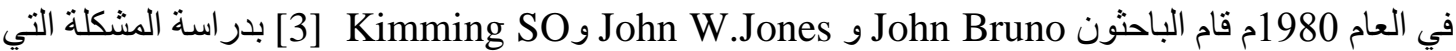
تحدث في أثناء القيام بعملية جدولة (Scheduling) مجمو عة من المهام (Tasks)، إما لمعالج مصدم بأسلوب خط الألناب النابيب

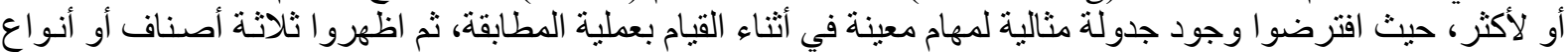

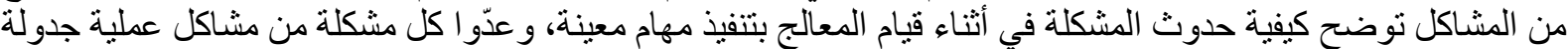

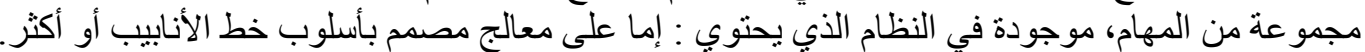

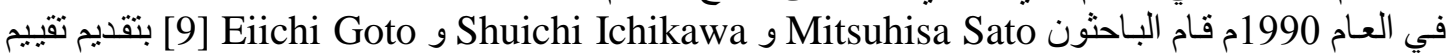

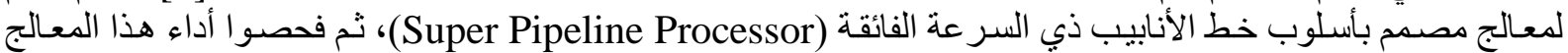

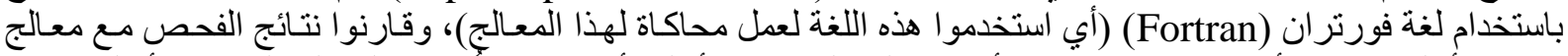

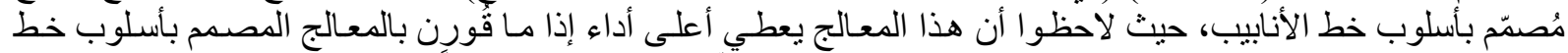

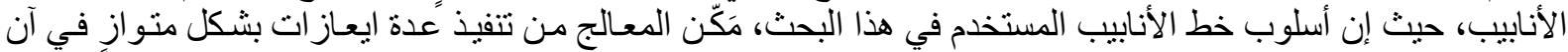

في العام 1993م قام الباحثان Jose M. Llaberia و Antonio M. Gonzalez [10 باستخدام آلية معينة

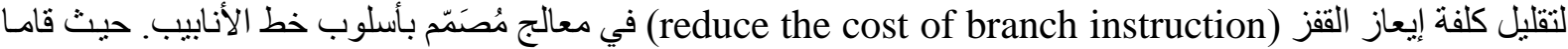

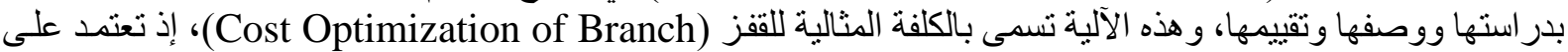

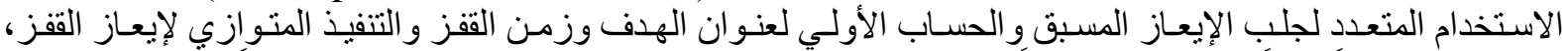

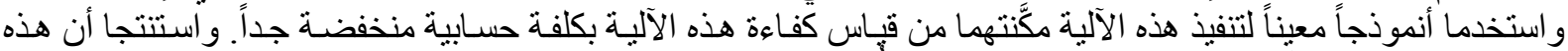

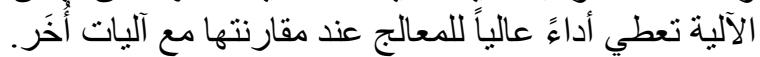

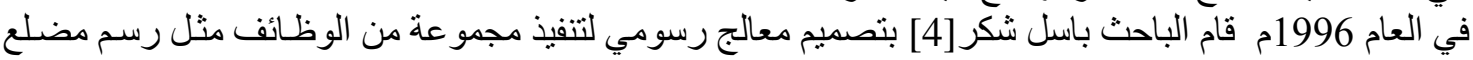

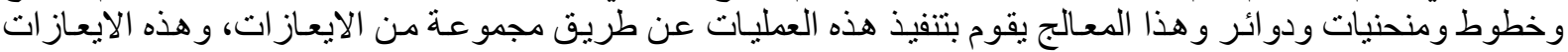

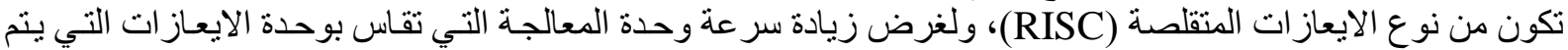

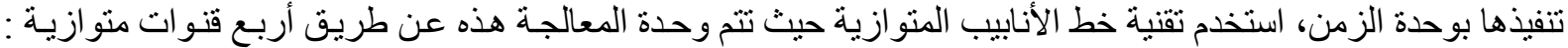

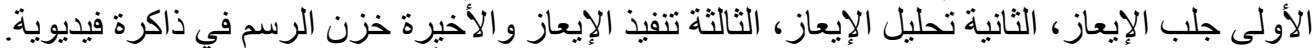

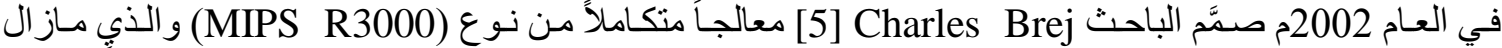

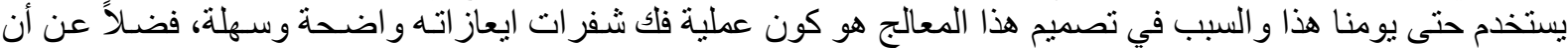
هذا النوع من المعالجات يحتوي على ذاكرة رئيسة (RAM) وذاكرة وسيطة (Cache) ودائرة لإدارة الذاكرة ( Control ونائة

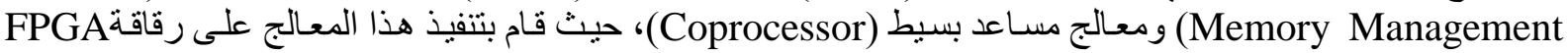
وفحص لبعض المكونات الرئيسة لهذا المعالج.

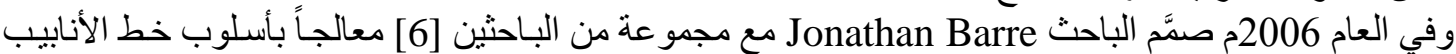

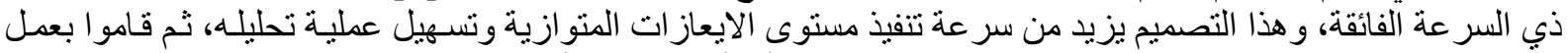

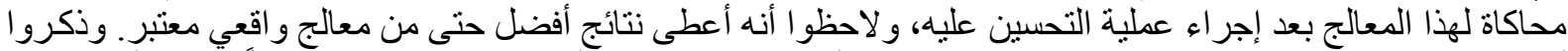

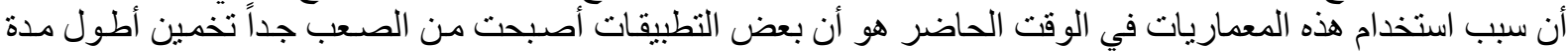

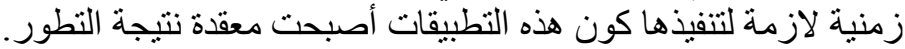

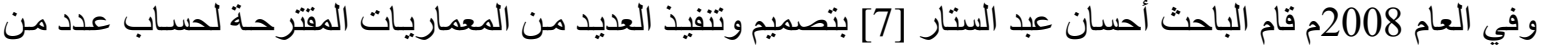

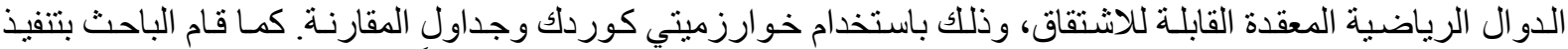

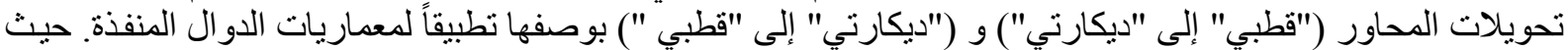

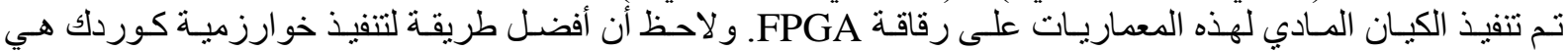
المعمارية المتو ازية بأسلوب خط لهذه الأنابيب. 


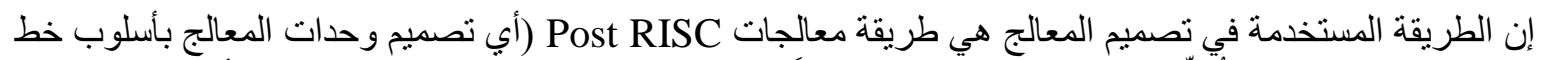

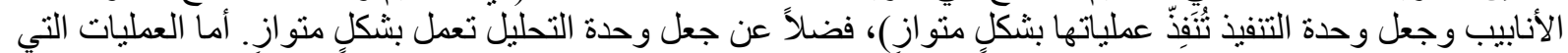

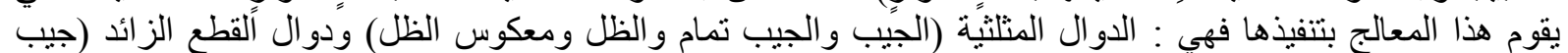

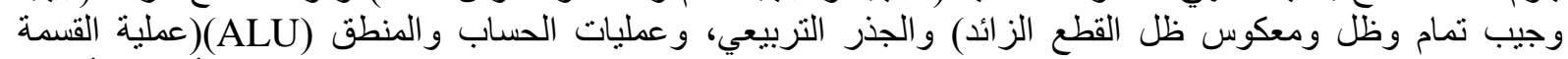

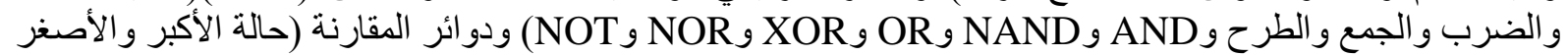

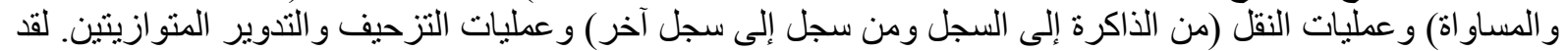

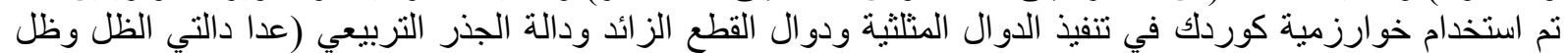

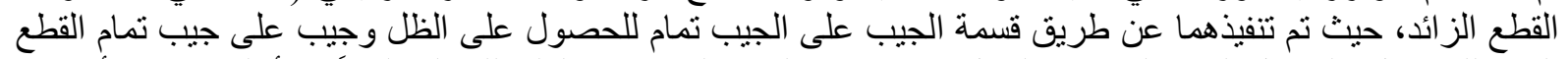

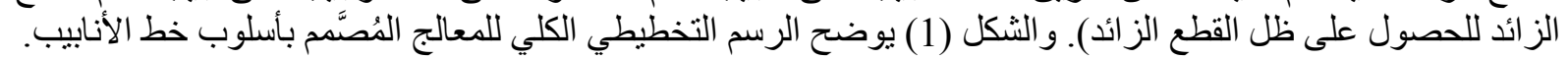

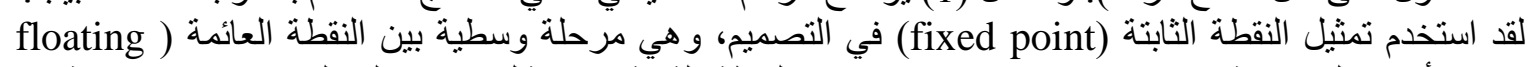

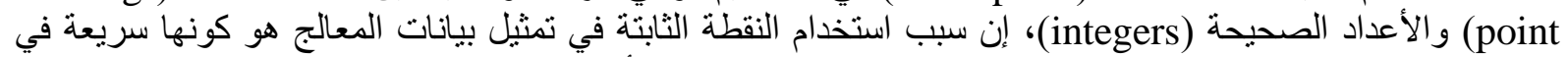

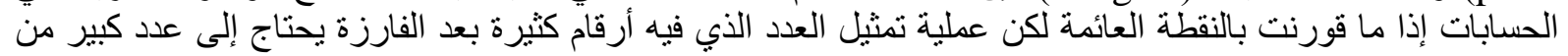

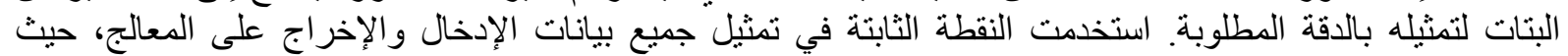

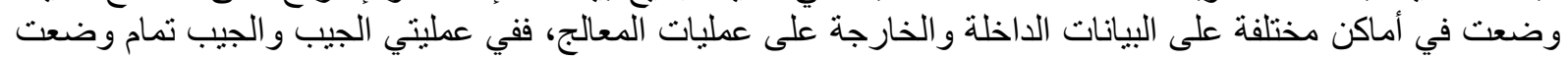

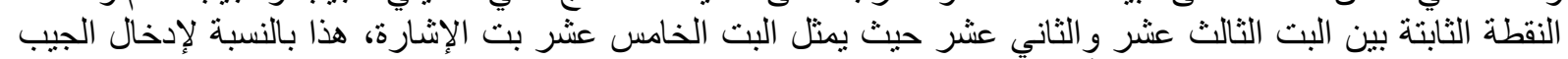

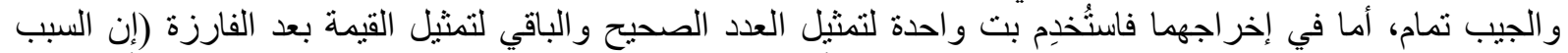

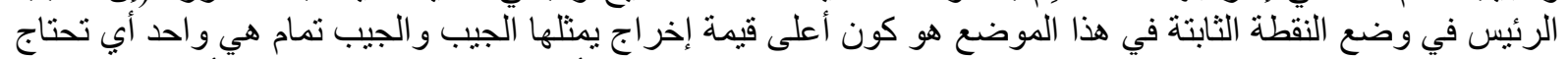

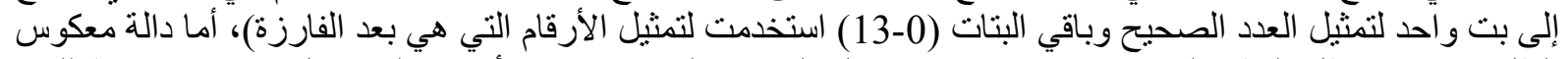

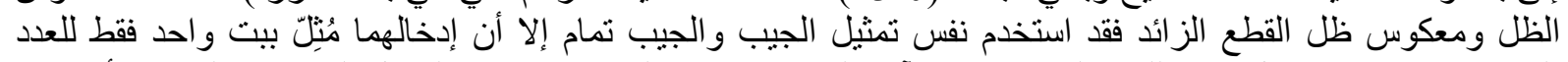

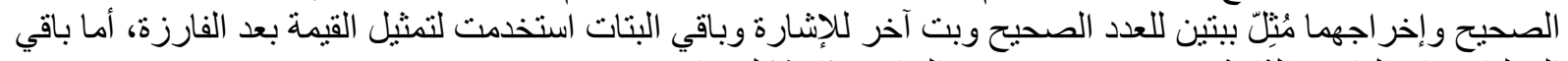

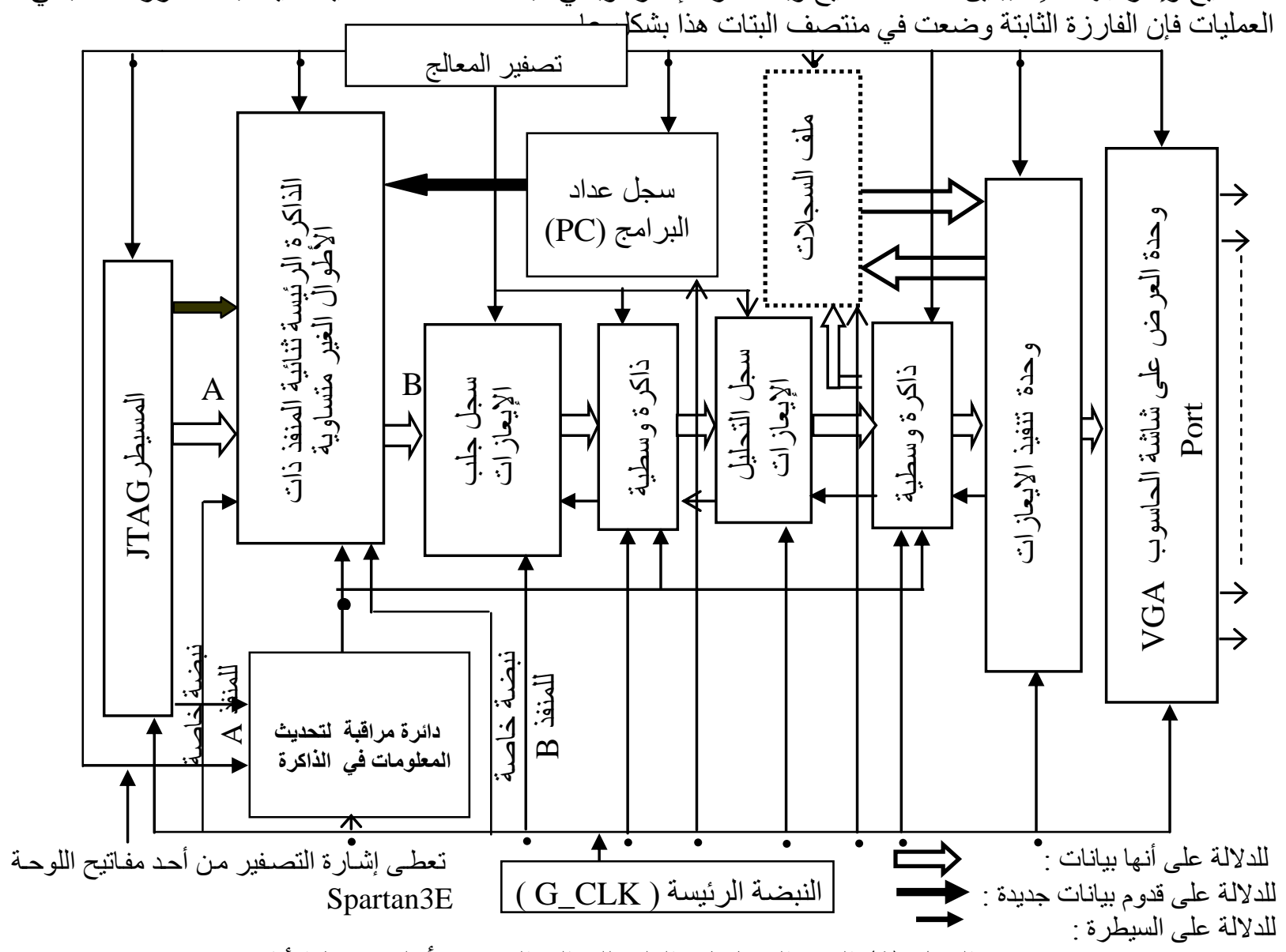

الثكل (1) الرسم التخطبطي الكلي للمعالج المصمم بأسلوب خط الأنابيب 


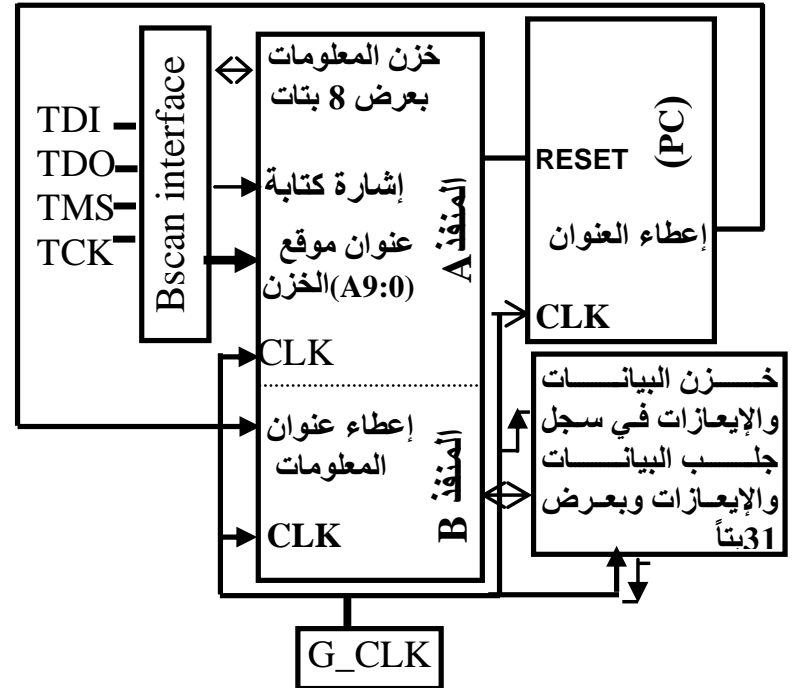

الثنكل (2) ربط المعالج مع الذاكرة الرئيسة وJTAG
: Main Memory) الأكرة الرئيسة

إن الذاكرة الرئيسة المستخدمة في هذا البحث هي

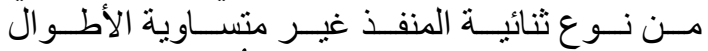
(mismatch length) (A التحديث البيانات و الإيعاز ات المخزونة فيها

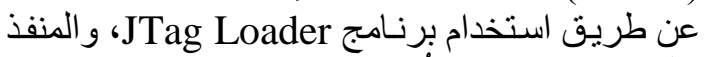

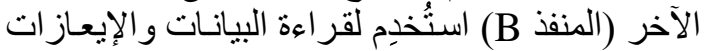

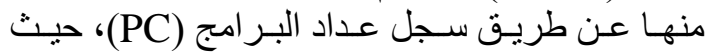

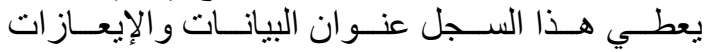

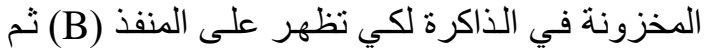
يقوم سجل جلب الإيعاز ات بخزنها في ذاكرنه المؤقتتة، و الثكل (2) يوضح ربط المعالج بالذاكرة الرئيسة.

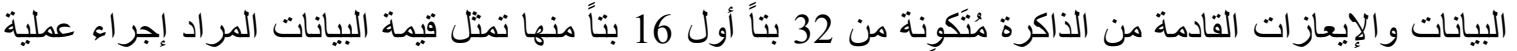

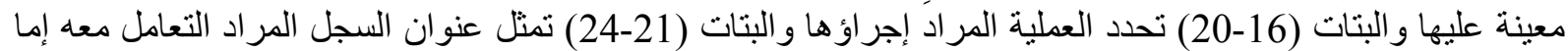

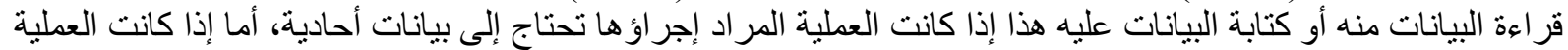

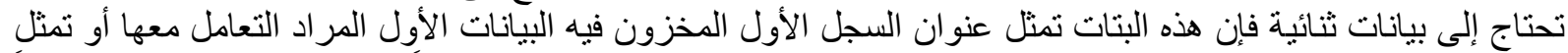

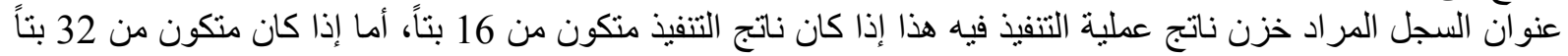

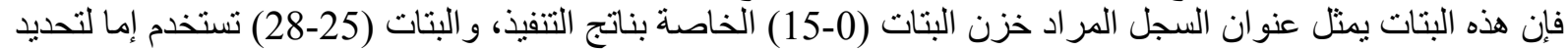

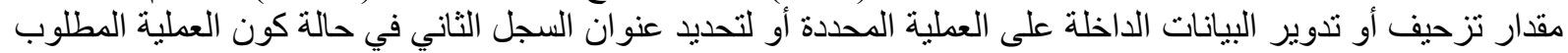

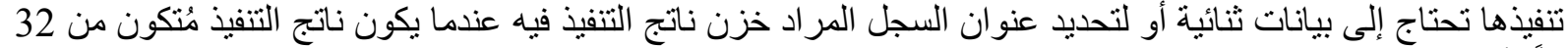

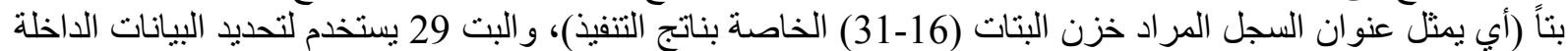

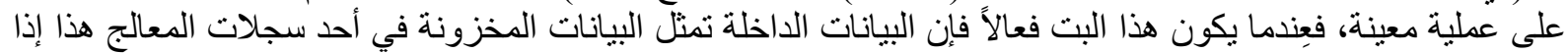

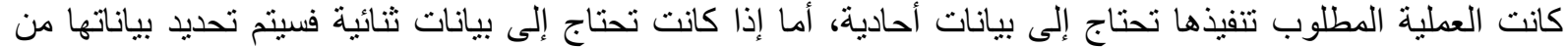

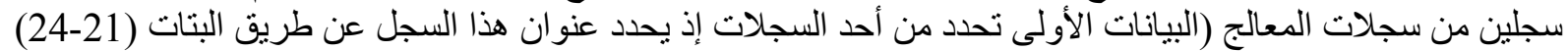

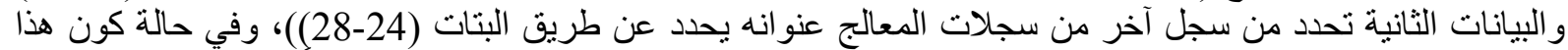

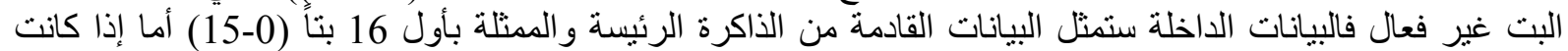

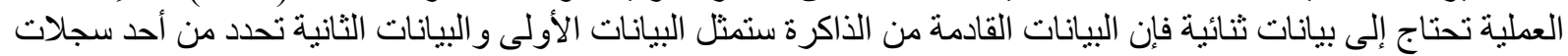

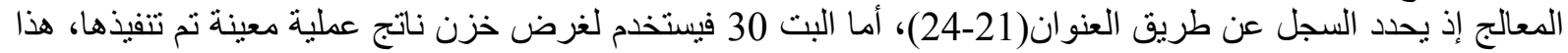

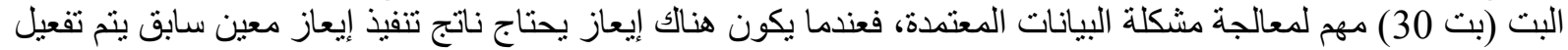

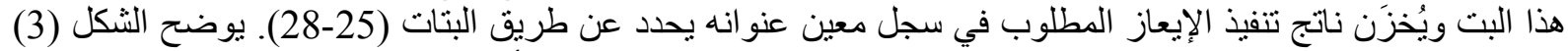

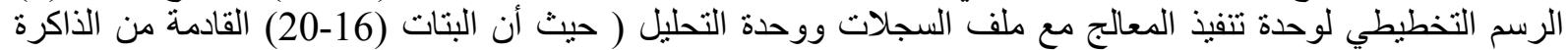

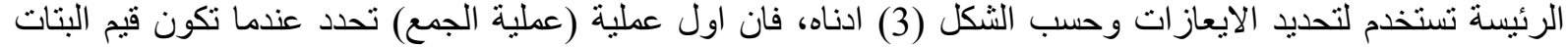

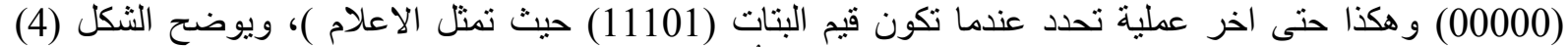
المخطط الانسيابي لعملية التحليل الكاملة للإيعاز وكيفية تحديد كُل عملية وبياناتها، بينما يوضح النيات النكل (5) الهيئة أو البنية العامة للإيعاز ات المسيطرة على البرنامج. 
محمود: تصميم معالج رياضي بأسلوب خط الأنابيب ومضاعفة السرعة وتنفيذه باستخدام FPGA

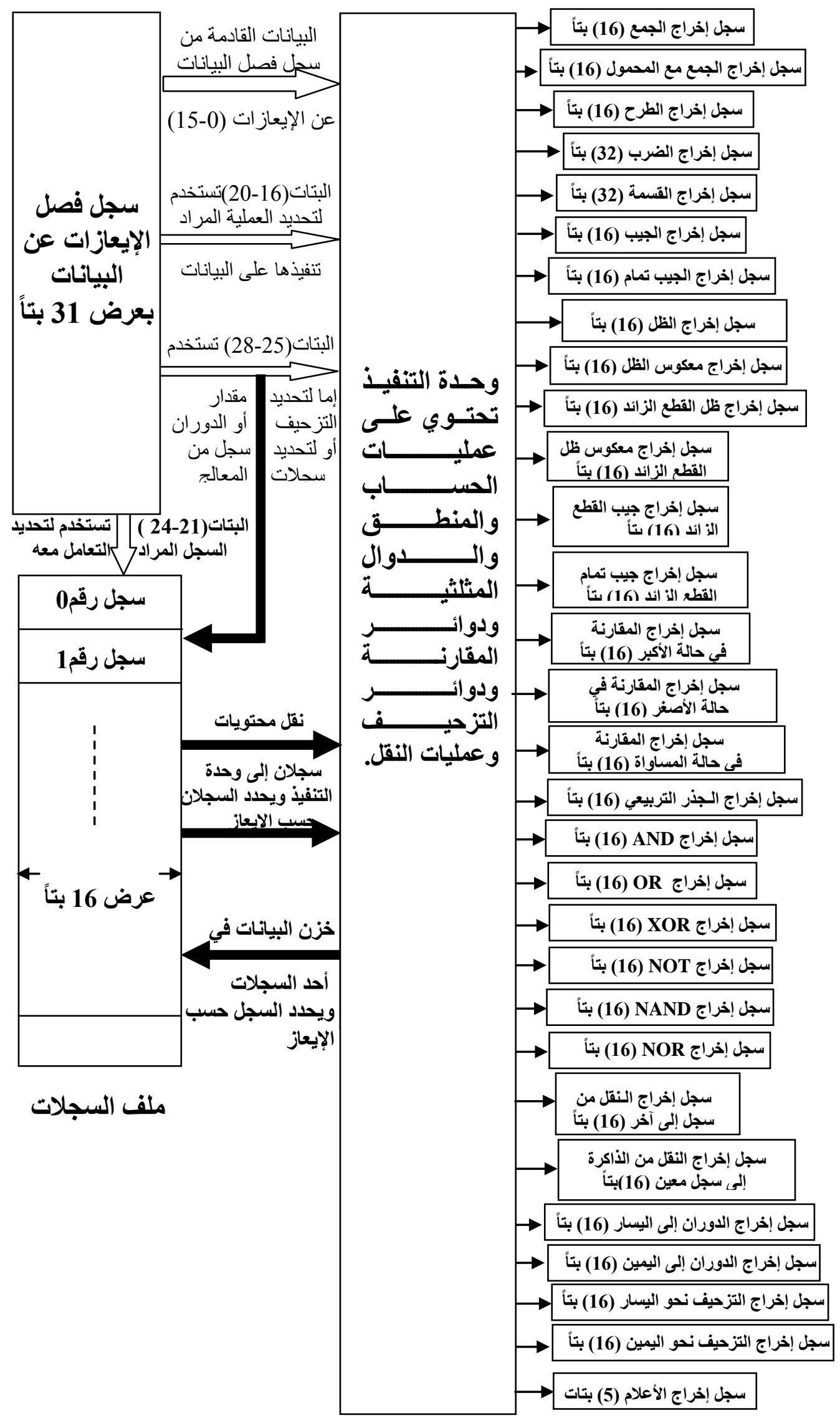

الثكل(3) الرسم التخطيطي لوحدة تنفيذ المعالج مع ملف السجلات ووحدة التحليل 

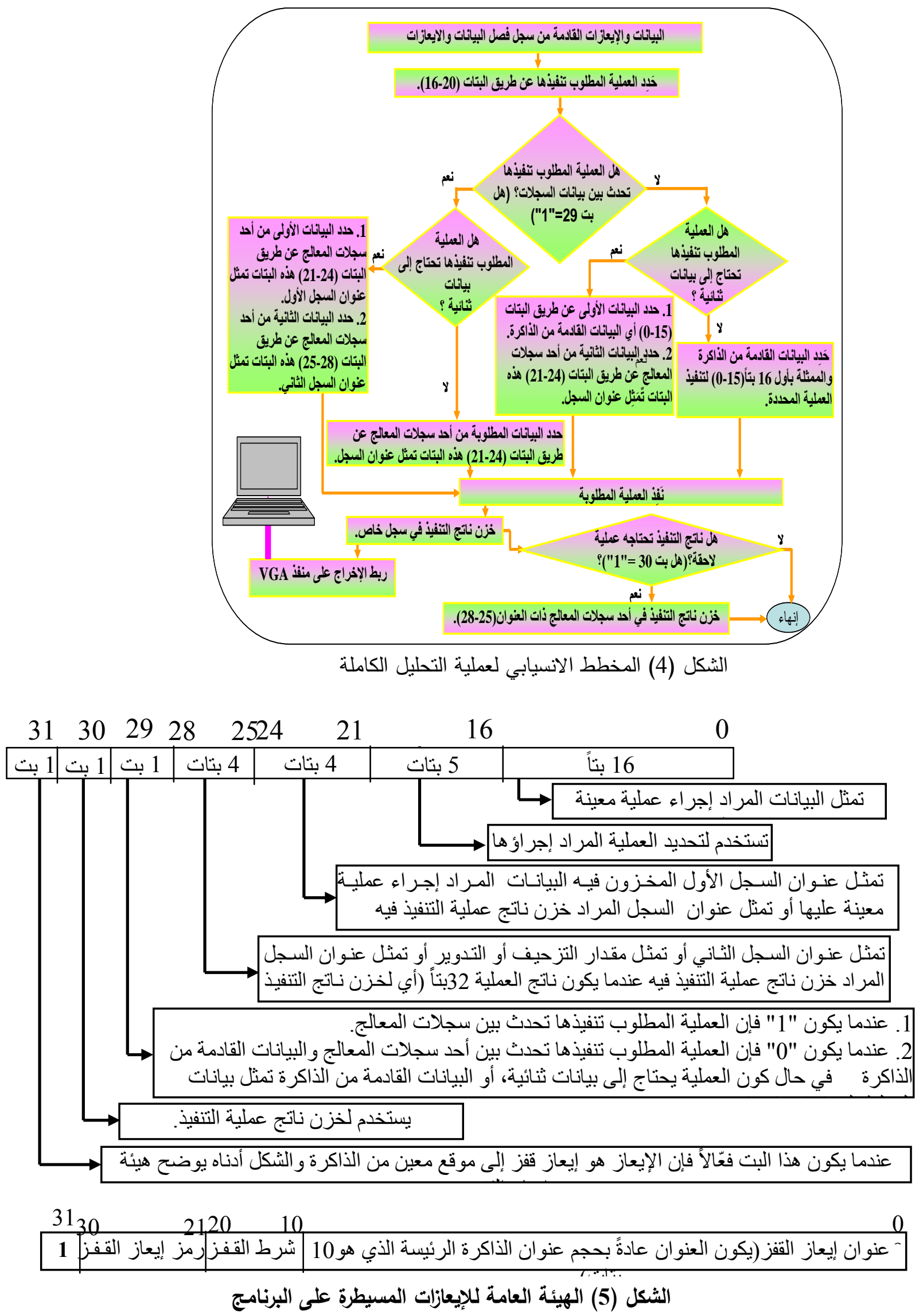


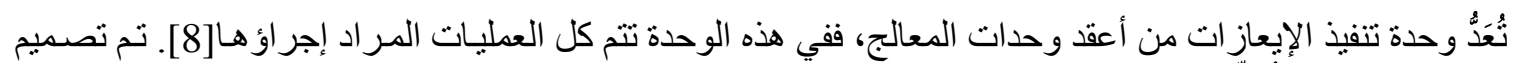

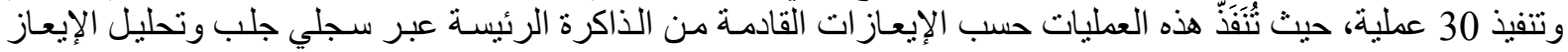

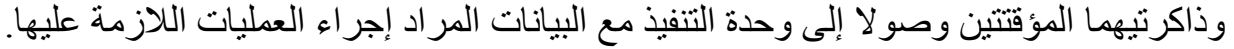

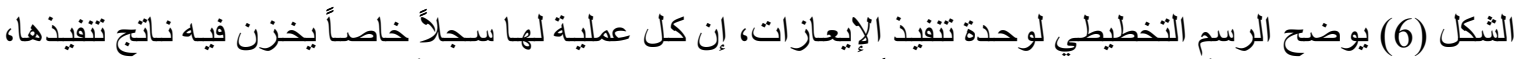

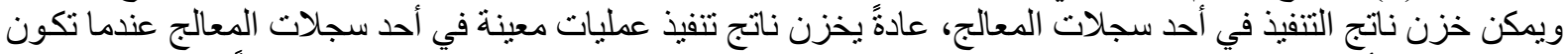

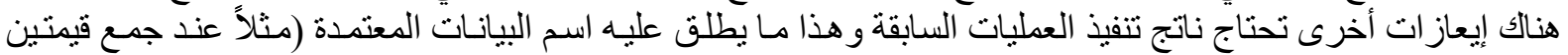
معينتين ثم ضرب ناتج الجمع في قيمة أخرى).

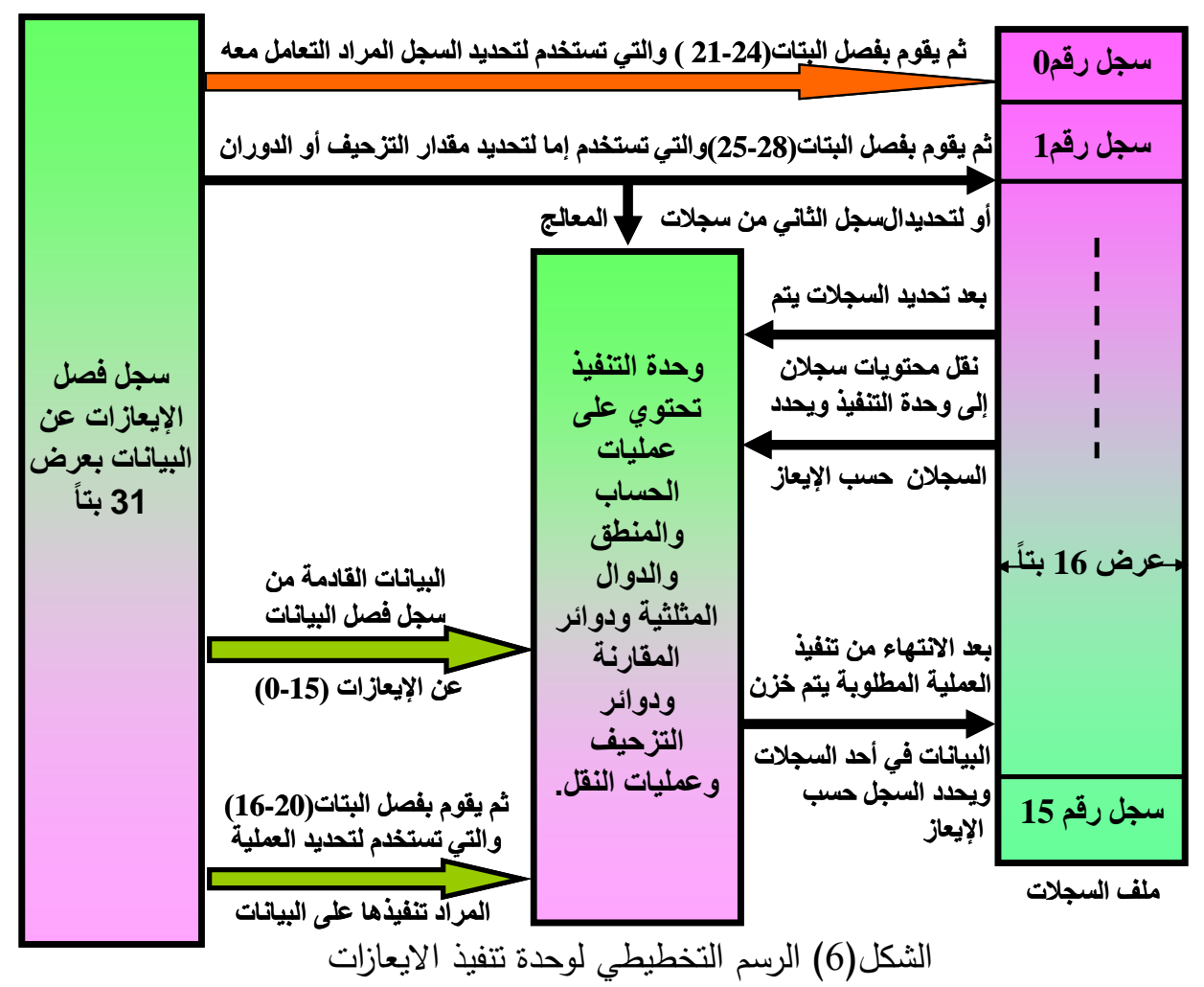

\section{مضاعفة عرض النبضة :}

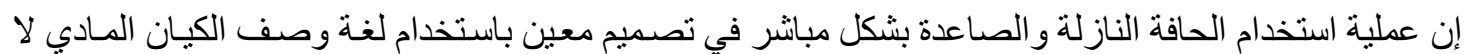

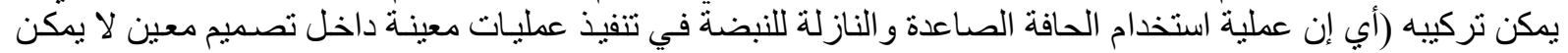

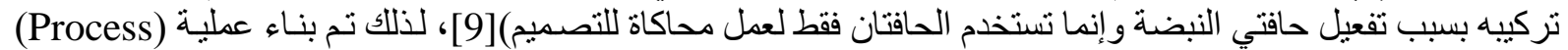

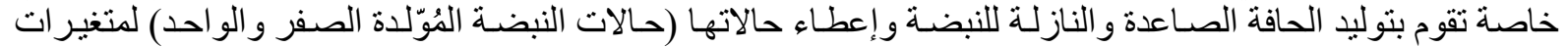

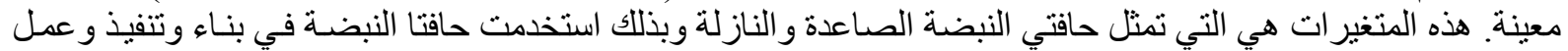

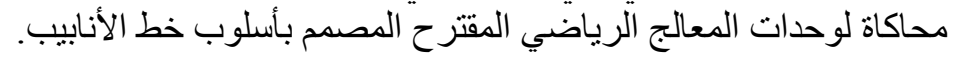

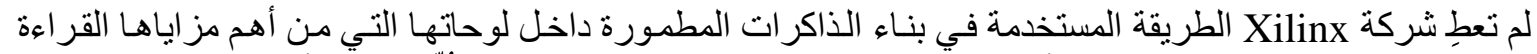

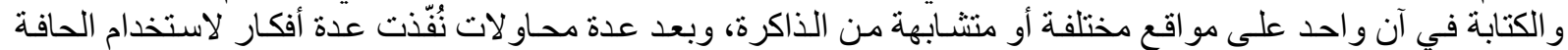

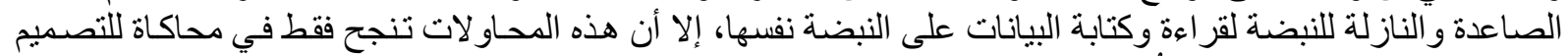

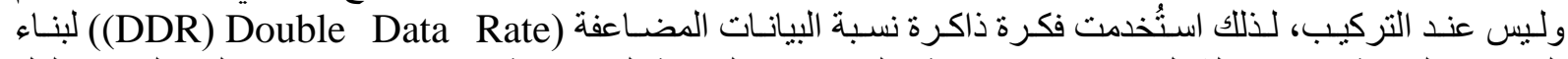

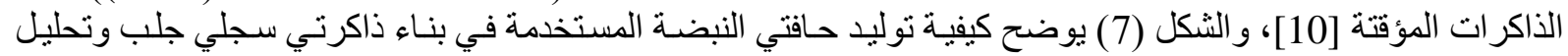

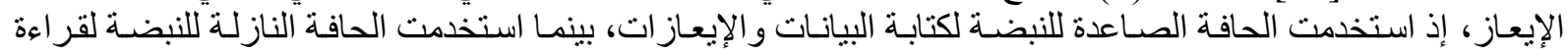




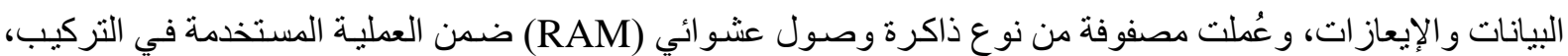

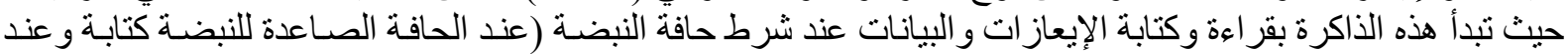

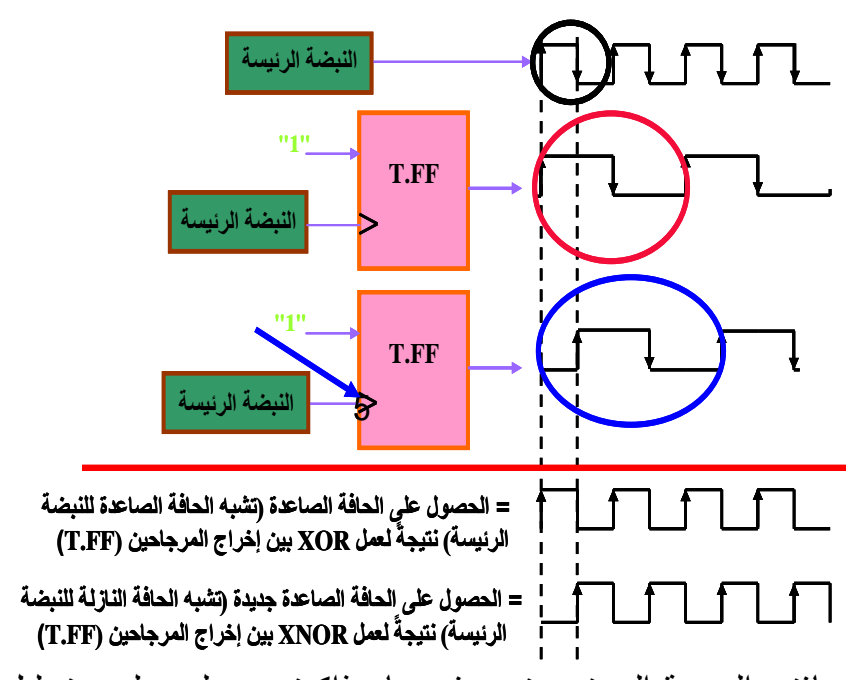

الحافة النازلة للنبضة قرة اءة).

الثكل (7) توليد حافتي النبضة المستخدمنين في بناء ذاكرتي سجلي جلب وتحليل الإيعاز

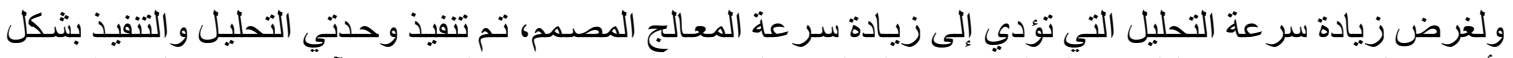

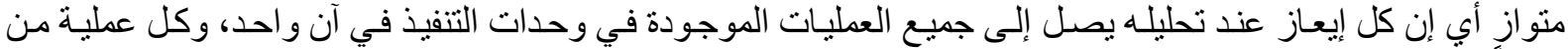

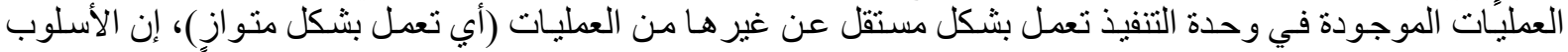

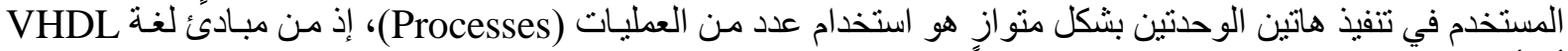

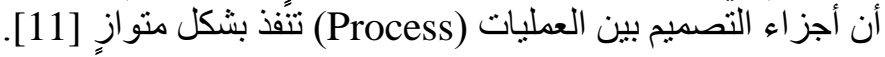

4.2 بناء وحدة التنفيذ :

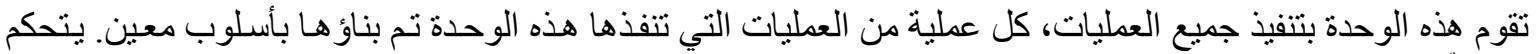

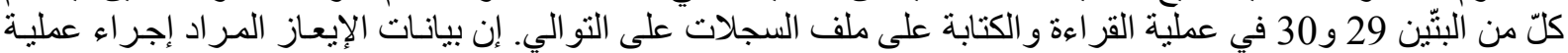

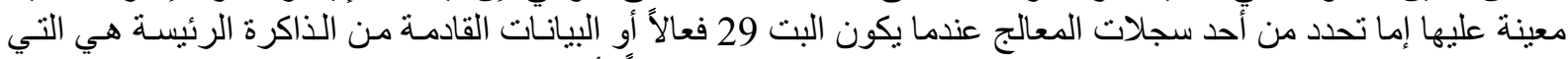

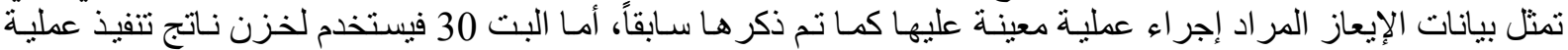

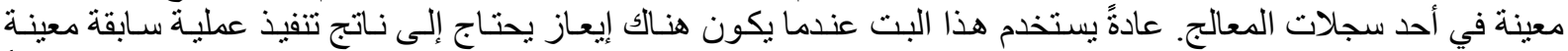

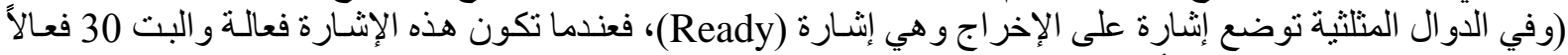

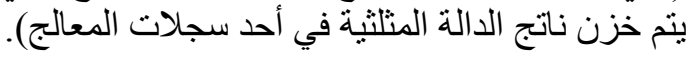

وفيما يأتي شرح عن بعض عمليات المعالج المصمم : 1.4.2 1 دالتا الجيب والجيب تمام :

تم تنفيذ كل من عمليتي الجيب و الجيب تمام بأسلوب خط الأنابيب باستخدام خو ارزمية كوردك، حيث تم استخدام لغنة

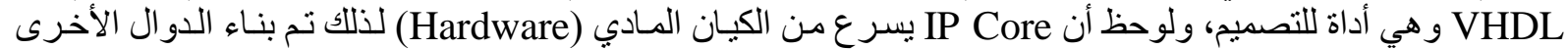

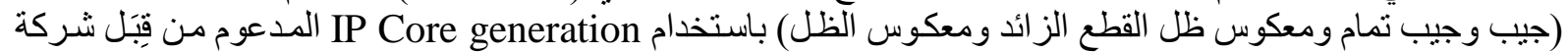

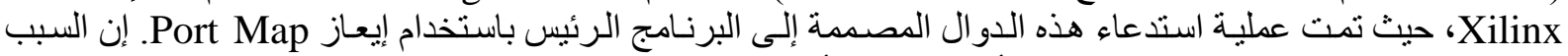

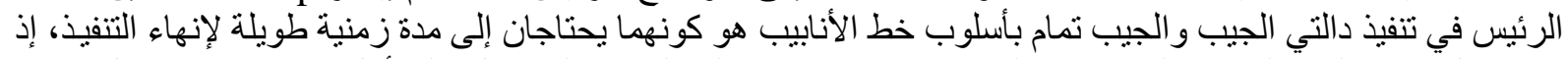

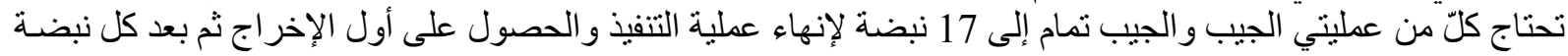

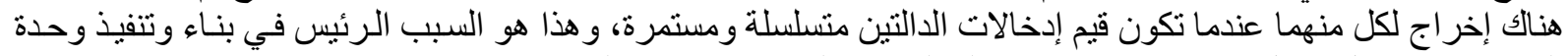

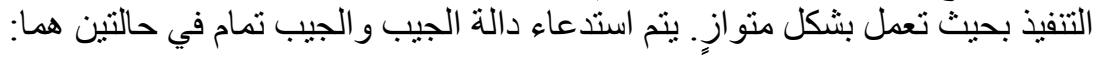

الأولى: عند القيام بتنفيذ دالتي الجيب و الجيب تمام في الحالة الاعتيادية.

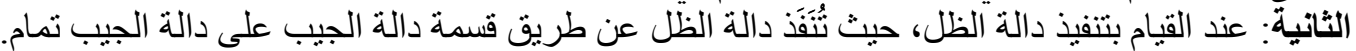


2.4.2

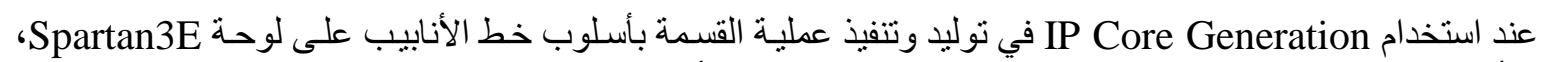

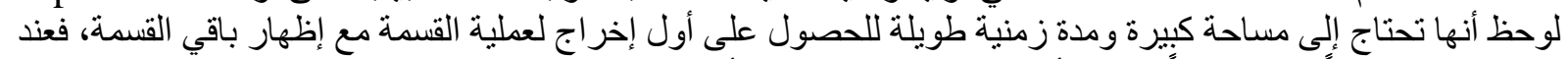

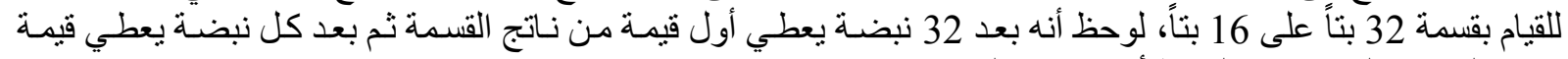

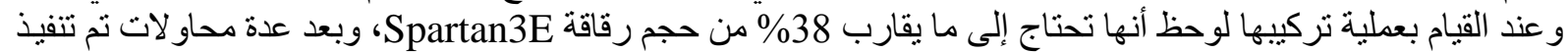

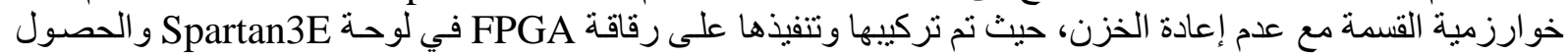

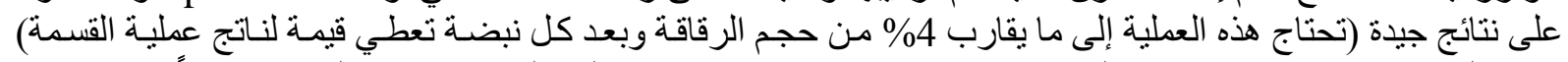

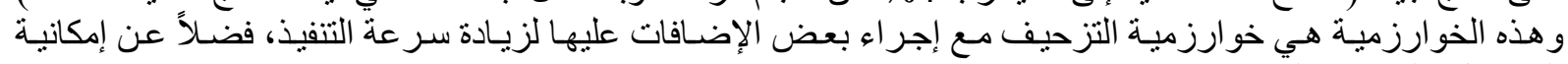
الحصول على باقي القسمة منها.

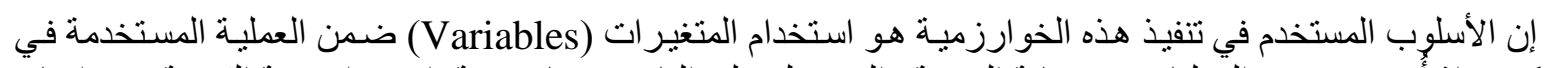

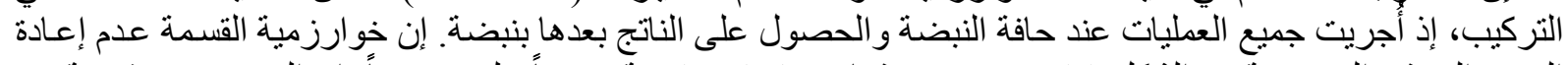

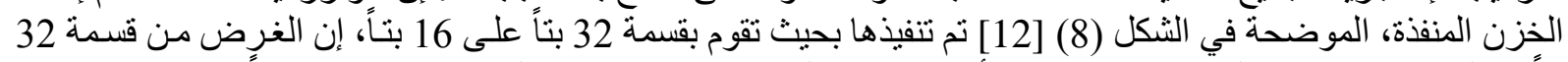

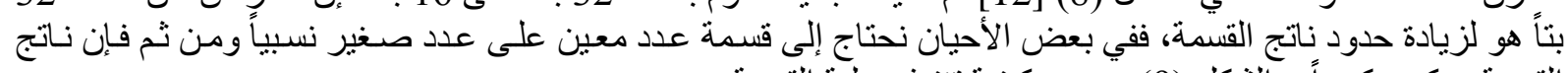

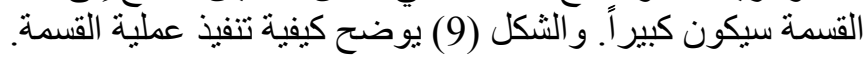

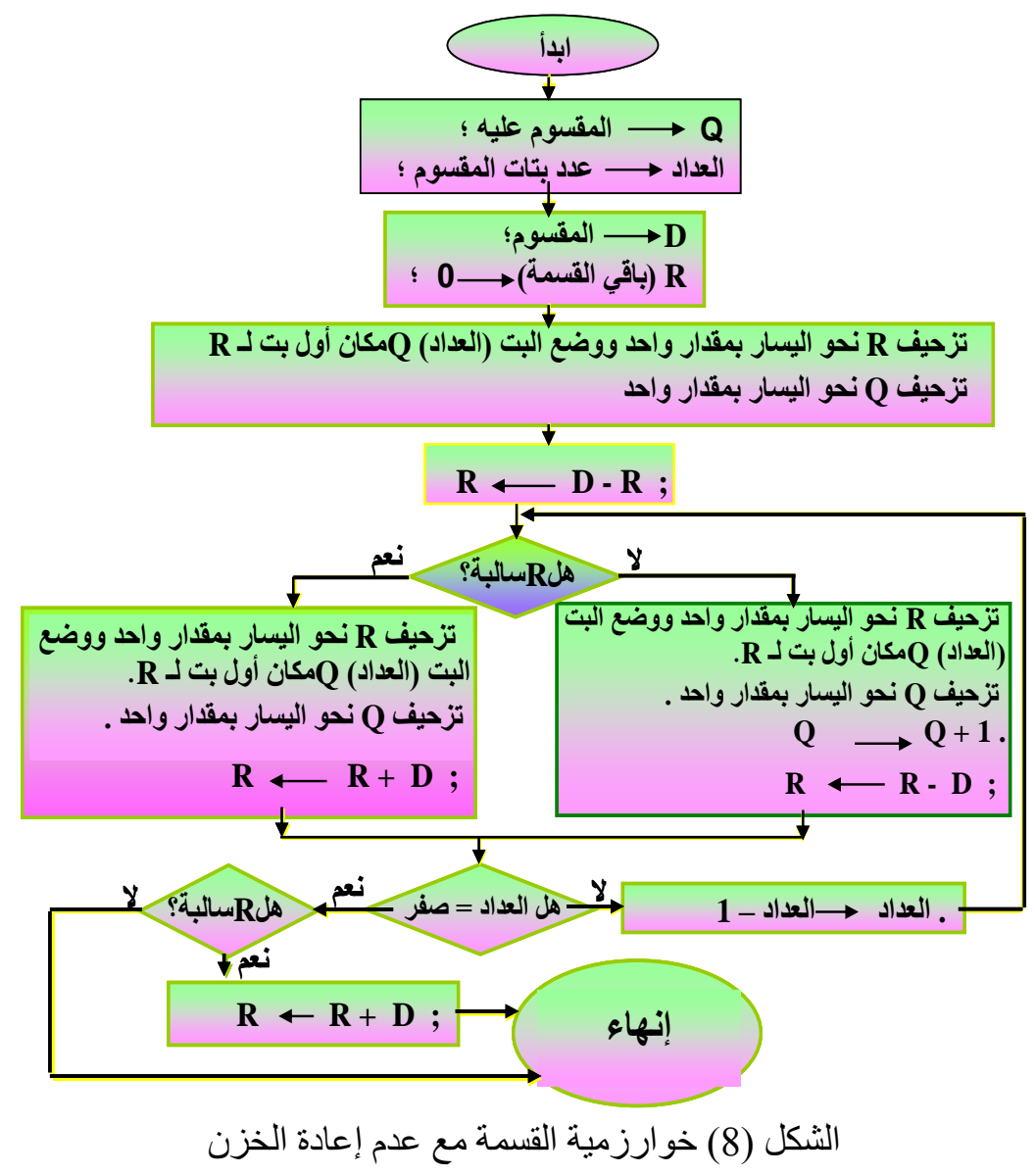

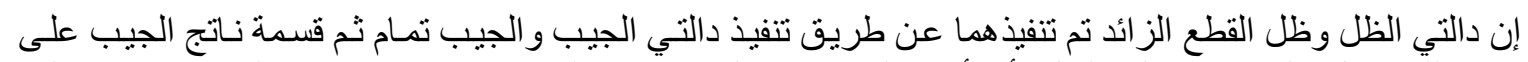

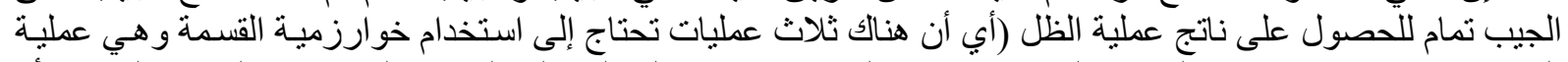

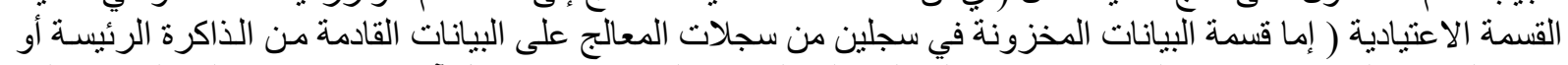

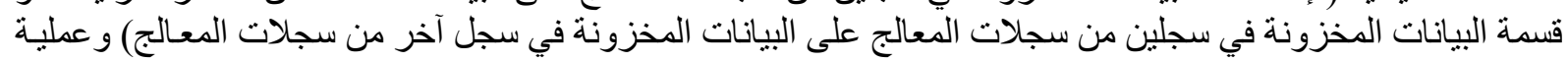

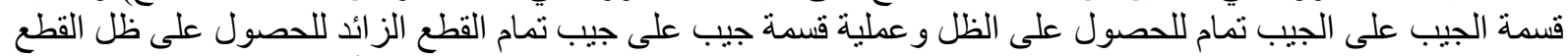

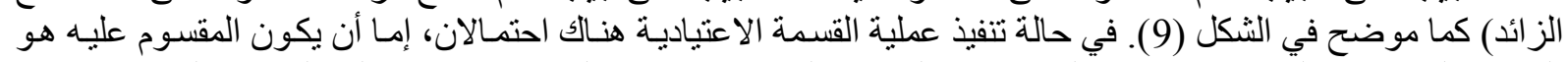

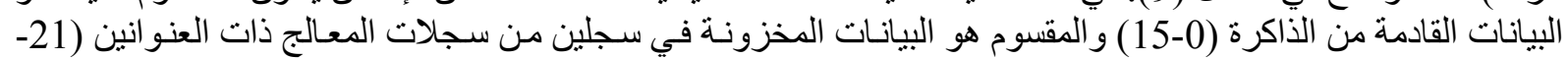




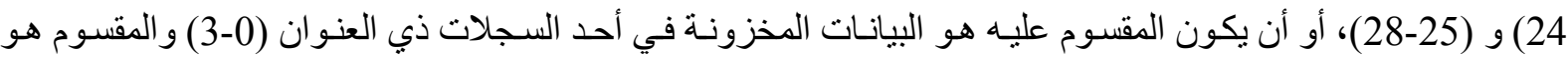

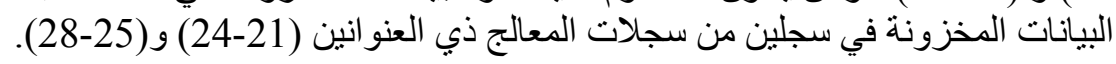

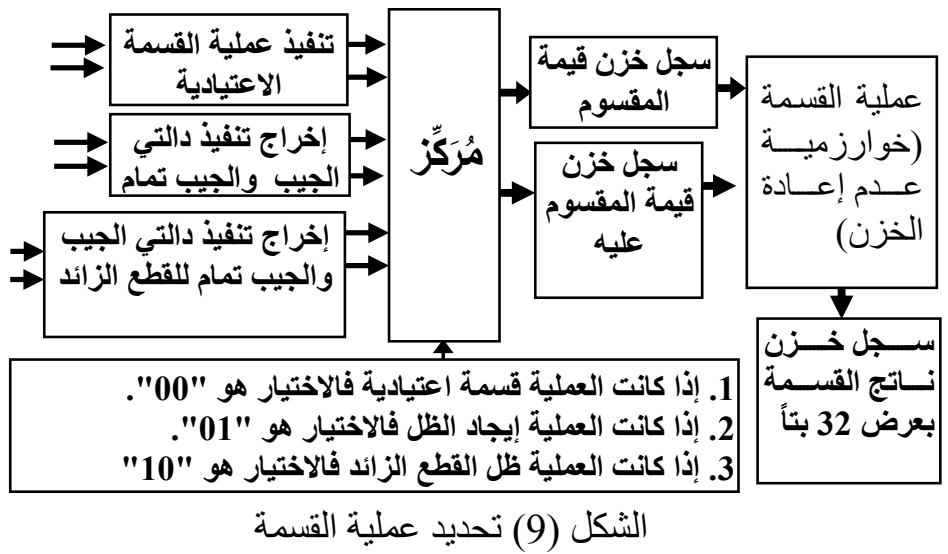

3.4.2 الجيب والجيب تمام للقطع الزائد والجذر التربيعي ومعكوسي الظل وظل القطع الزائد:

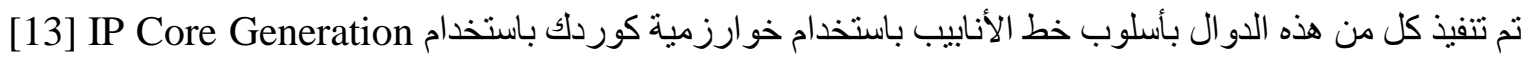

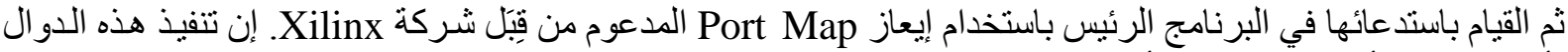
بأسلوب خط الأنابيب يزيد من الأداء و السر عة و الثنكل (10) يوضح تنفيذ دالئ دالة معكوس الظل.

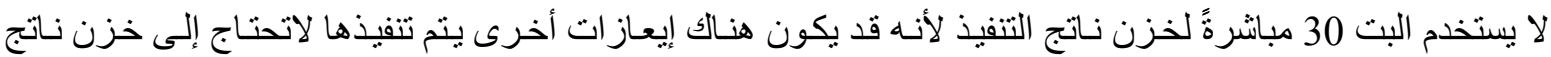

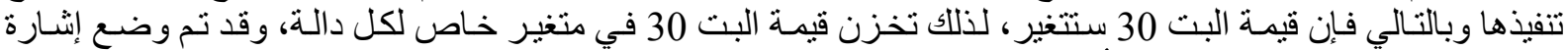

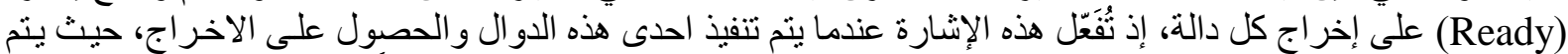
عمل ضرب منطقي بين إثنارة Ready و المتغير الخاص بالدالة المعنية، فإذا كان الناتج واحداً فيخزن ناتج التنفيذ في سجل التهل

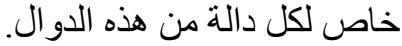

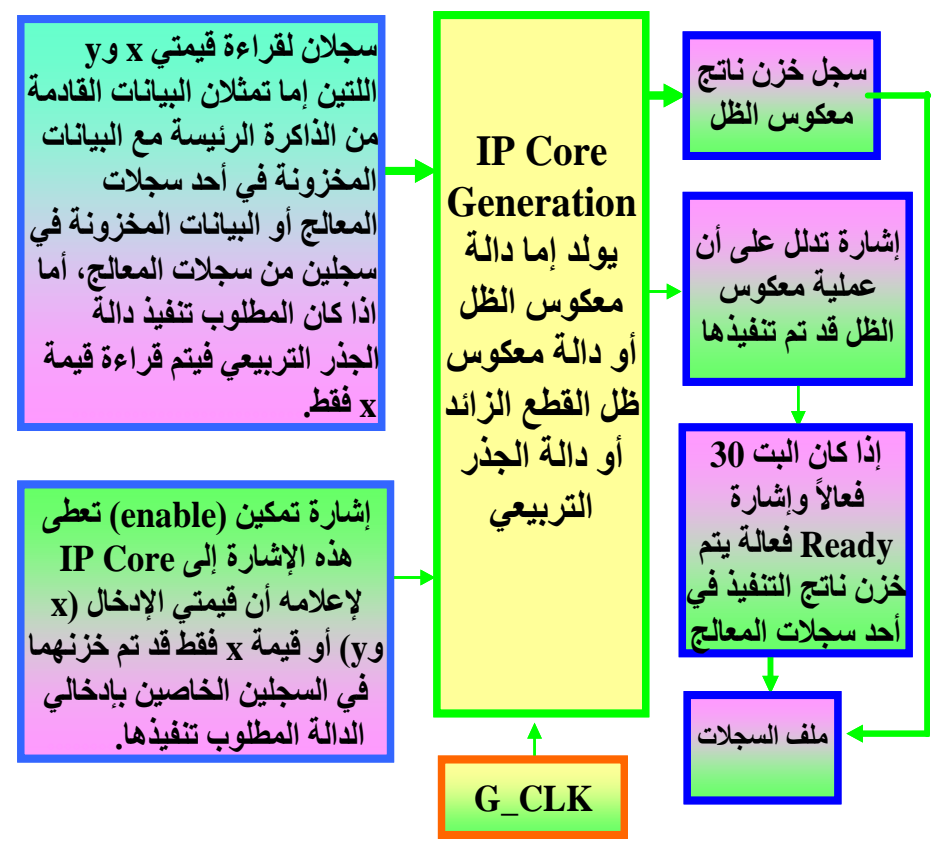

الثكل (10) عملية معكوس الظل المنفذ بأسلوب خط الأنابيب 
محمود: تصميم معالج رياضي بأسلوب خط الأنابيب ومضاعفة السرعة وتنفيذه باستخدام FPGA

\subsubsection{0}

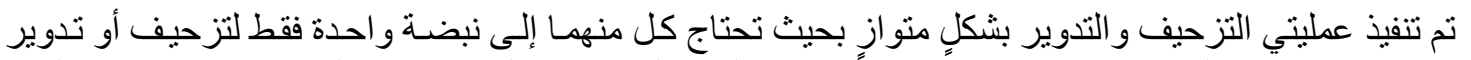

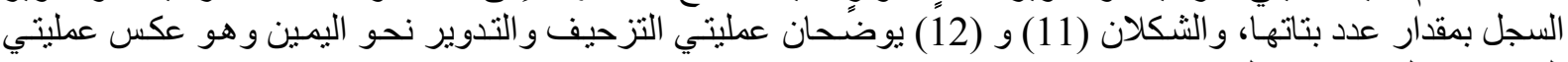
التزحيف و التدوير نحو اليسار.
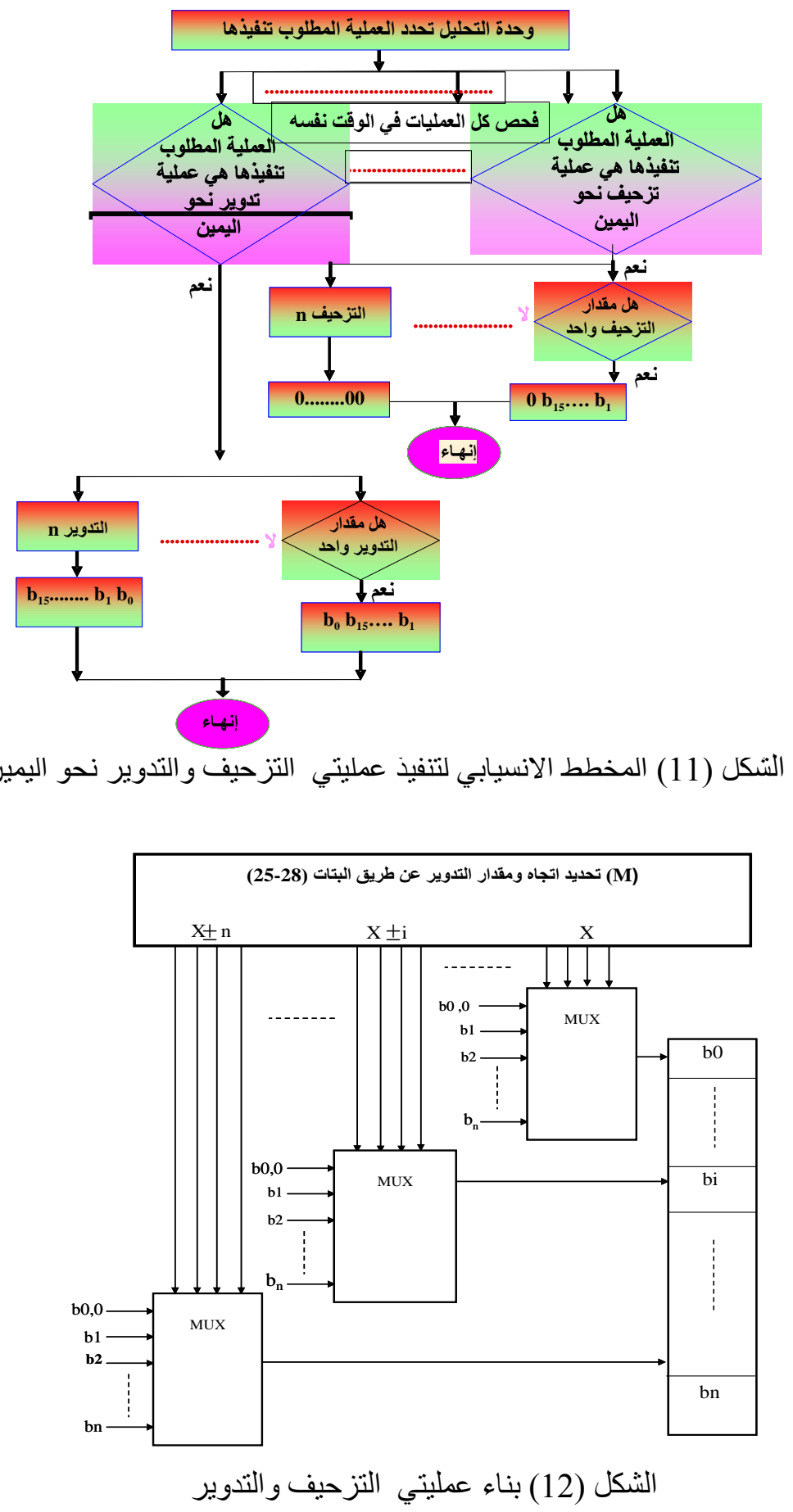
: Flags) 5.4.2

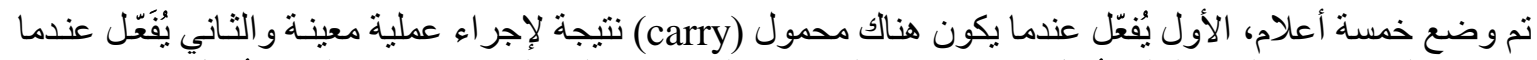

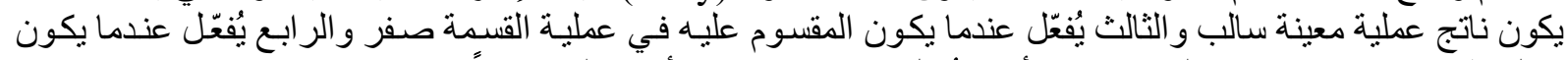

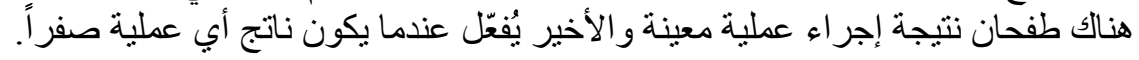

3.

Xتضمن هذه النتائج، عرض نتائج المحاكاة لبعض العمليات التي يقوم المعالج بتنفيذها باستخدام الأداة Simulator (Test Bench) على شاشنة الحاسوب.

1.3 كميات الموارد المستخدمة في تنفيذ المعالج :

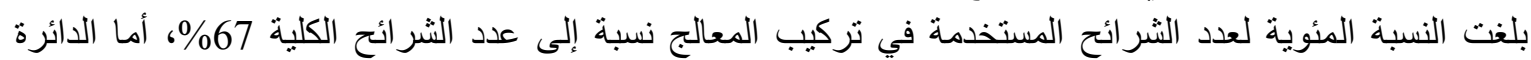

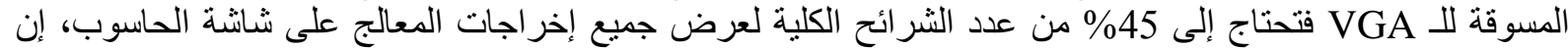

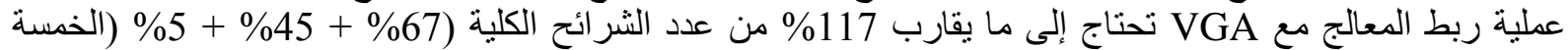

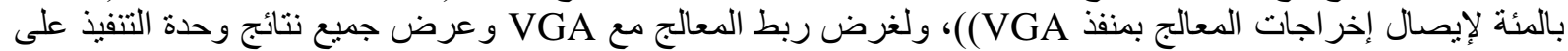

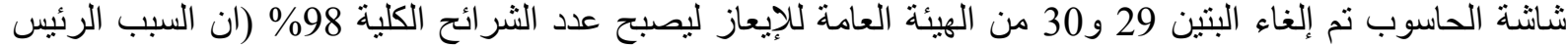

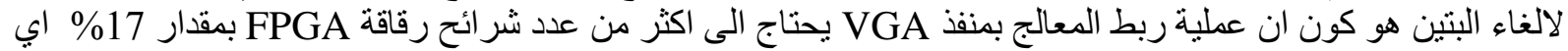

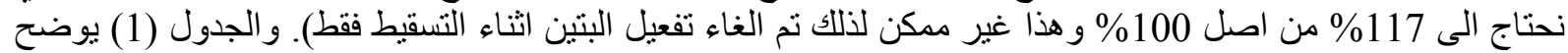

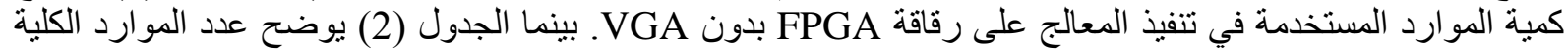

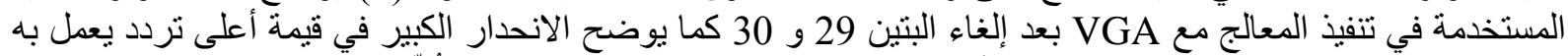

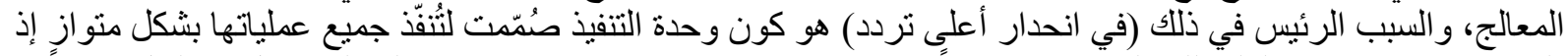

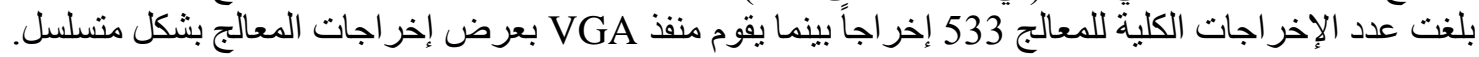

الجدول (1) الموارد المستخدمة في الكيان المادي لتنفيذ المعالج بدون VGA.

\begin{tabular}{|l|c|c|c|}
\hline \multicolumn{1}{|c|}{ Type Resources } & $\begin{array}{c}\text { Utilized } \\
\text { Resources }\end{array}$ & $\begin{array}{c}\text { Total } \\
\text { Resources }\end{array}$ & Ratio \\
\hline N. of Slices & 3010 & 4656 & $\% 64$ \\
\hline N. F.Flops & 5354 & 9312 & $\% 57$ \\
\hline N. of 4 i/p LUTs & 5408 & 9312 & $\% 58$ \\
\hline N. of IOBs & 2 & 232 & $\% 0$ \\
\hline N. of Block RAMS & 3 & 20 & $15 \%$ \\
\hline N. of GCLKs & 2 & 24 & $8 \%$ \\
\hline Op. Freq. & \multicolumn{3}{|c|}{$133.820 \mathrm{MHz}$} \\
\hline
\end{tabular}

الجدول (2) الموارد المستخدمة في الكيان المادي لتنفيذ المعالج مع VGA.

\begin{tabular}{|l|c|c|c|}
\hline \multicolumn{1}{|c|}{ Type Resources } & $\begin{array}{c}\text { Utilized } \\
\text { Resources }\end{array}$ & $\begin{array}{c}\text { Total } \\
\text { Resources }\end{array}$ & Ratio \\
\hline N. of Slices & 4587 & 4656 & $\% 98$ \\
\hline N. F.Flops & 5955 & 9312 & $\% 63$ \\
\hline N. of 4 i/p LUTs & 8345 & 9312 & $\% 89$ \\
\hline N. of IOBs & 16 & 232 & $\% 6$ \\
\hline N. of Block RAMS & 4 & 20 & $\% 20$ \\
\hline N. of GCLKs & 3 & 24 & $\% 12$ \\
\hline Op. Freq. & \multicolumn{3}{|c|}{$11.301 \mathrm{MHz}$} \\
\hline
\end{tabular}


عند القيام بتركيب المعالج، لوحظ أن ذاكرتي جلب وتحليل الإيعاز ات بنيتا بشكل ذاكرتين كتليتين، علماً أن كلاً منههـا

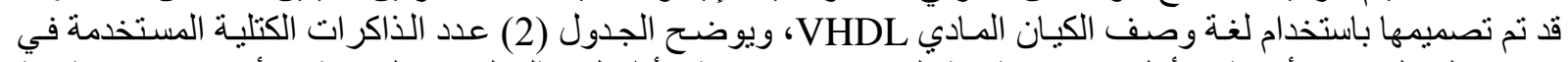

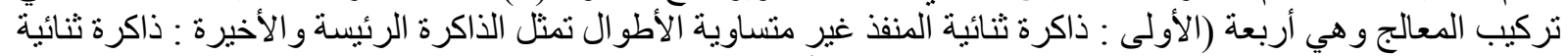

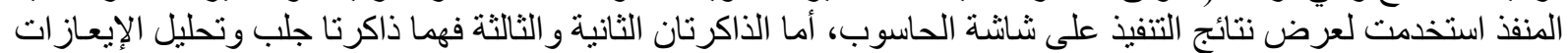
اللتان صممتا باستخدام لغة وصف لتفان الكيان المادي).

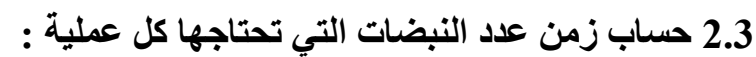

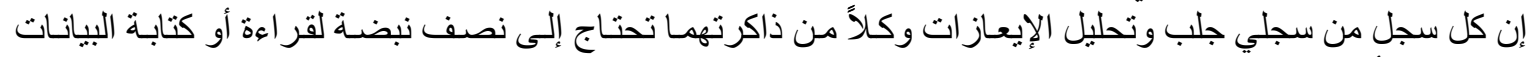

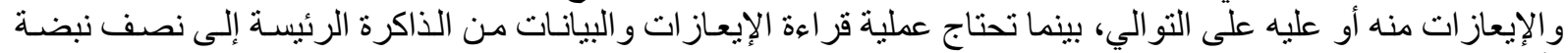

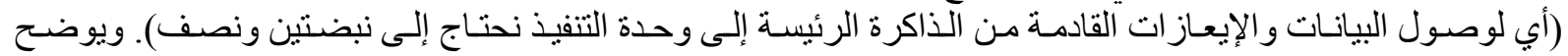

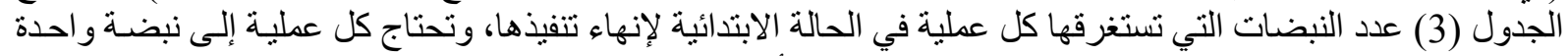

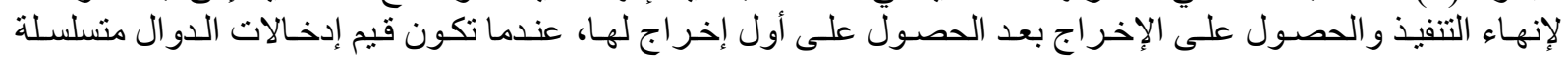

ومستمرة.

الجدول (3) عدد النبضات التي تحتاجها كل عملية لإنهاء التنفيذ في الحالة الابتدائية حيث M :هي عدد النبضات

\begin{tabular}{|c|c|}
\hline عدد النبضـات لتنفيذ N من المهام & العمليات \\
\hline $\mathrm{M}=\mathrm{N}$ & 1. عمليات الحساب و المنطق و عمليات التدوير و التزحيف و عمليات النقل. \\
\hline $\mathrm{M}=\mathrm{N}+17$ & 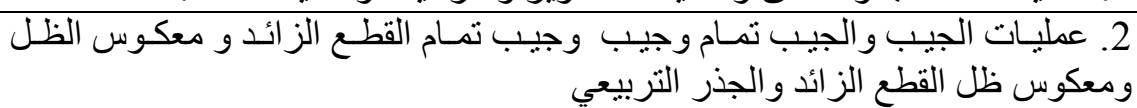 \\
\hline$M=N+23$ & 3. عمليتي الظل وظل القطع الز ائد \\
\hline
\end{tabular}

• محتويات سجلات المعالج في الحالة الابتدائية :

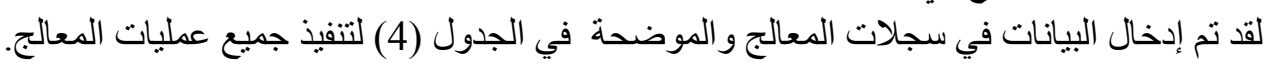

الجدول (4) القيم الابتدائية المخزونة في ملف السجلات

\begin{tabular}{|c|c|c|c|c|c|c|c|c|}
\hline 7 & 6 & 5 & 4 & 3 & 2 & 1 & 0 & السجل \\
\hline FF01 & 0AA0 & 9999 & $\overline{\mathrm{A016}}$ & FFF1 & 011F & $\mathrm{C893}$ & $5 \mathrm{CC5}$ & محتوياته \\
\hline $\mathbf{F}$ & $\mathbf{E}$ & D & $\mathbf{C}$ & B & $\mathbf{A}$ & 9 & 8 & السجل \\
\hline $376 \mathrm{C}$ & D2BE & 2D41 & 0000 & E000 & 376C & 00FA & 1FFF & محتوياته \\
\hline
\end{tabular}

3.3 عرض نتائج المحاكاة لبعض العمليات :

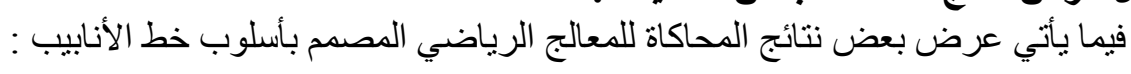
(5)

تمت عملية حساب المعادلة أعلاه وحساب عدد النبضات التي تحتاجها لإنهاء التنفيذ إذ تم تحويل و إيجاد ناتج كل عملية

$(0.25)_{\mathrm{D}}=(2000)_{\mathrm{H}} \quad,\left(\frac{\pi}{3}\right)_{\mathrm{D}}=(2182)_{\mathrm{H}}$ في المعادلة أعلاه وكما يلي:

$(0.5)_{\mathrm{D}}=(4000)_{\mathrm{H}}$

${ }^{(5)} \mathrm{D}=(0005)_{\mathrm{H}} \rightarrow 00310005 \rightarrow$ MOV R1,0005

$\left({ }_{\operatorname{SIN}} \frac{\pi}{3}\right)_{\mathrm{D}}=(376 \mathrm{D}) \mathrm{H} \rightarrow 40452182 \rightarrow$ SIN R2,2182

$\left(5-\sin \frac{\pi}{3}\right) \mathrm{D}=(\mathrm{C} 898) \mathrm{H} \rightarrow 64220000 \rightarrow$ SUB R1,R2

$(\sqrt{0.25}=0.5) \rightarrow 40692000 \rightarrow$ SR R3,2000 
$(\sqrt{0.25}+5)_{\mathrm{D}}=(4005)_{\mathrm{H}} \rightarrow 62610000 \rightarrow \mathrm{ADD} \mathrm{R} 3, \mathrm{R} 1$

$\left.{ }^{(4005)}\right)^{*}(\mathrm{C} 898)_{\mathrm{H}}=(3229 \mathrm{EAF} 8)_{\mathrm{H}} \rightarrow 22630000 \rightarrow$ MUL R3,R1

بلغت عدد النبضات اللازمة لإنهاء التنفيذ والحصول على الإخراج النهائي للمعادلة أعلاه 23 نبضة حيث كان الناتج

النهائي للحصول على القيمة النهائية للمعادلة هو (3229EAF8)

$$
\text { : 1.3.3 محاكاة عمليتي التزحيف والتدوير : }
$$

لقد تم إدخال البيانات الآتية في الذاكرة الرئيسة و الموضحة في الجدول (5) و الثكل (13) يوضح نتائج تنفيذ عمليتي الجدول (5) نتائج حساب عمليتي التدوير والتزحيف

\begin{tabular}{|c|c|c|c|}
\hline العملية & 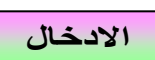 & مقدار التزحيف او التدوير & الناتج \\
\hline$\overline{\text { SHL }}$ & FOOF & 4 & OOFO \\
\hline SHR & ABCD & 1 & 55E6 \\
\hline ROL & ABCD & 2 & AF36 \\
\hline ROR & AOAO & 1 & 5050 \\
\hline
\end{tabular}

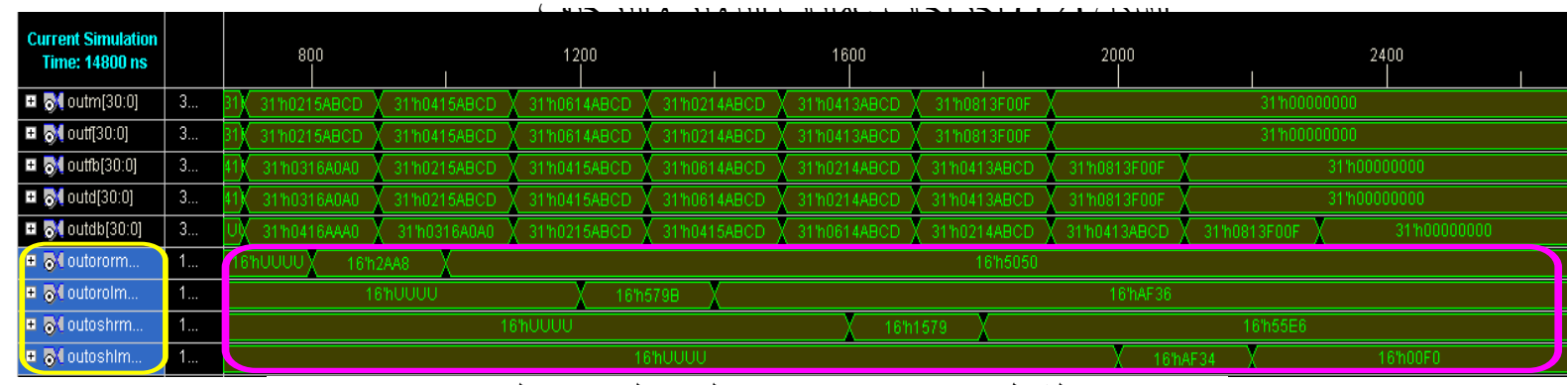

الثكل ( 13 ) ) إخر اجات عمليات التدوير و التزحيف

2.3.3 محاكاة عمليات الحسب :

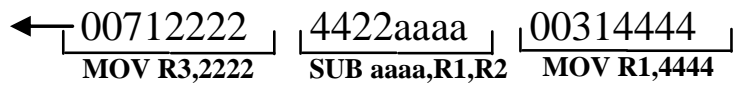

$\underset{\text { DIV R2,R4,FFFE }}{\stackrel{0484 f f f e}{\text { MUL R2,R4,R2,R4 }}} \frac{68430000}{4 \text { ADD 5555,R3,R4 }}$

البيانات و الإيعاز ات أعلاه استخدمت لحساب المعادلة أدناه و الثكل (14) يوضح ناتج محاكاة المعادلة أعلاه.

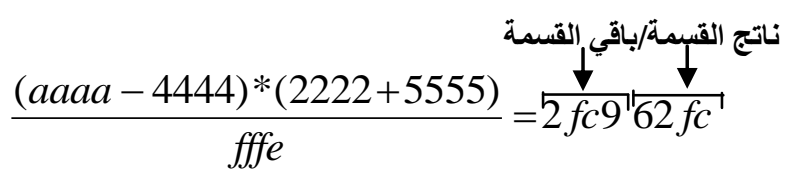


محمود: تصميم معالج رياضي بأسلوب خط الأنابيب ومضاعفة السرعة وتنفيذه باستخدام FPGA

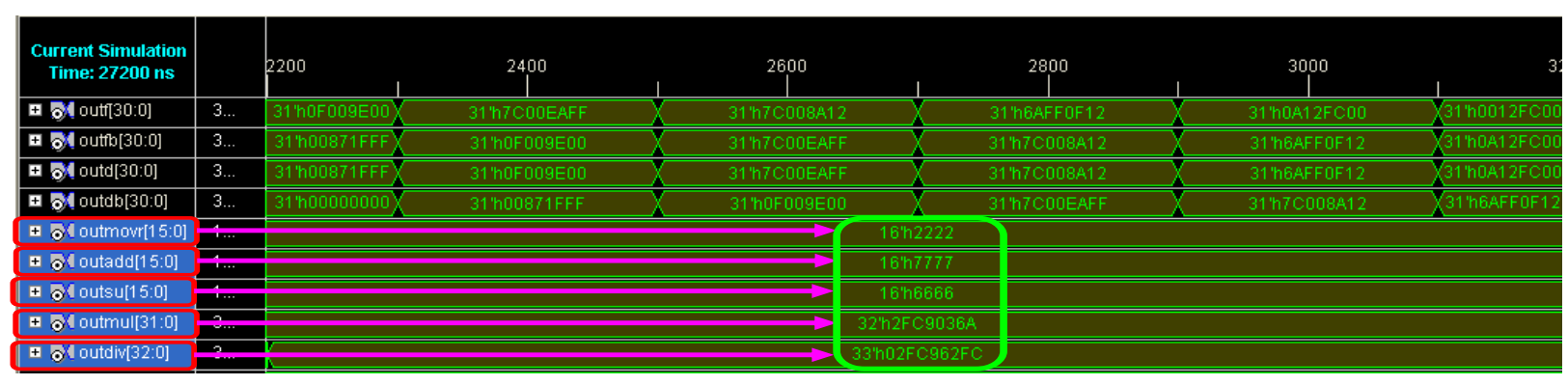

$$
\text { الثكل (14) ناتج المحاكاة لعمليات الجمع و الطرح و الضرب و القسمة }
$$

3.3.3

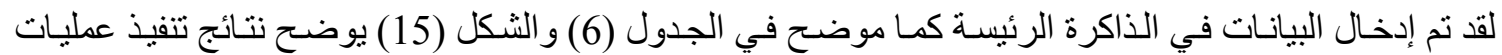

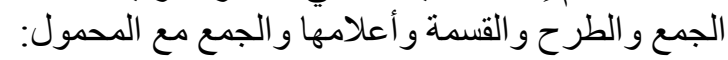
جدول (6) عملية الجمعو الطر حو القسمة القال

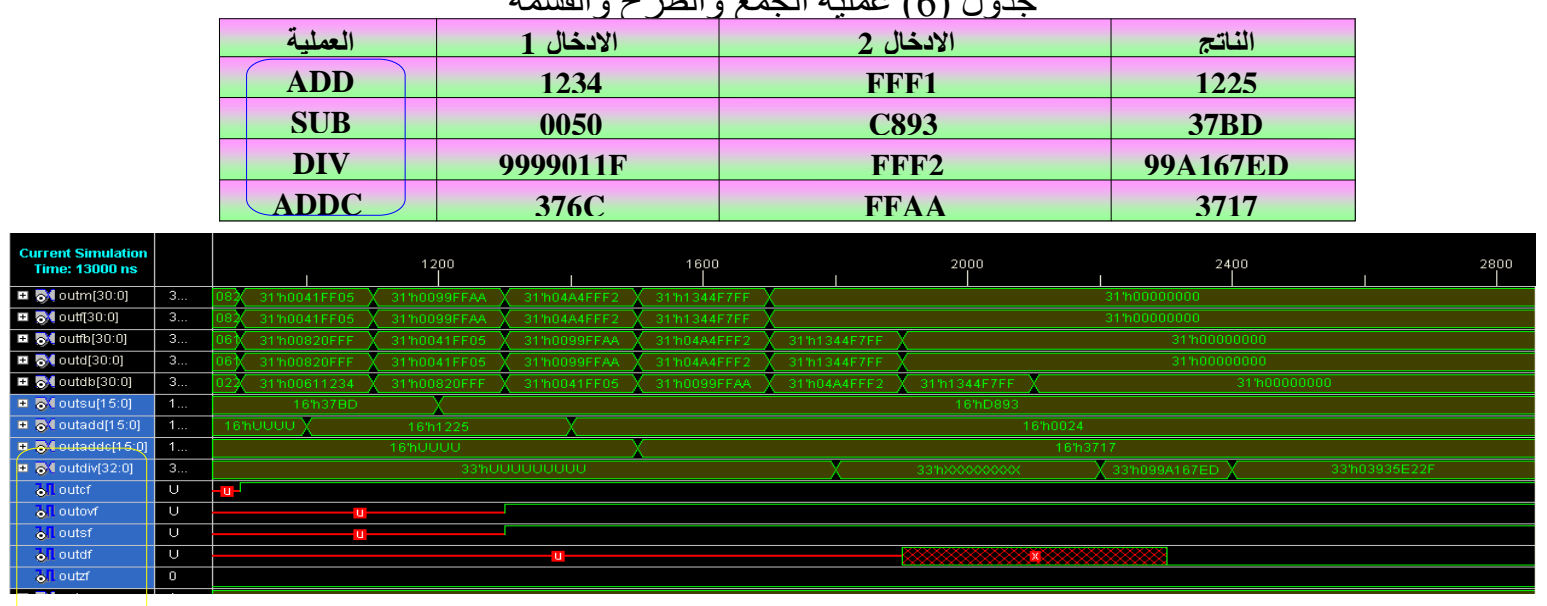

الثكل (15) يوضح نتائج محاكاة الاعلام

4.3.3 محاكاة دالة معكوس الظل ومعكوس ظل القطع الزائد ودالة الجذر التربيعي:

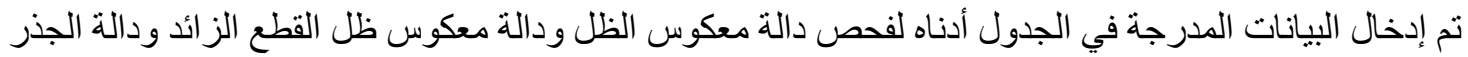

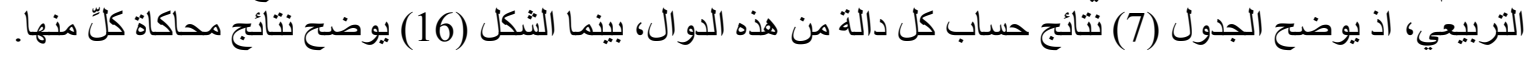

\begin{tabular}{|c|c|c|c|}
\hline في الذيم المخزونة & جيب القطع & جيب تمام القطع & الزاوية \\
\hline $00871 \mathrm{fff}$ & $376 C$ & 1fff & 2182 \\
\hline $00281 \mathrm{fff}$ & C893 & 1 ffff & DC38 \\
\hline 0009E000 & \multicolumn{2}{|c|}{ E000 } & A954 \\
\hline
\end{tabular}

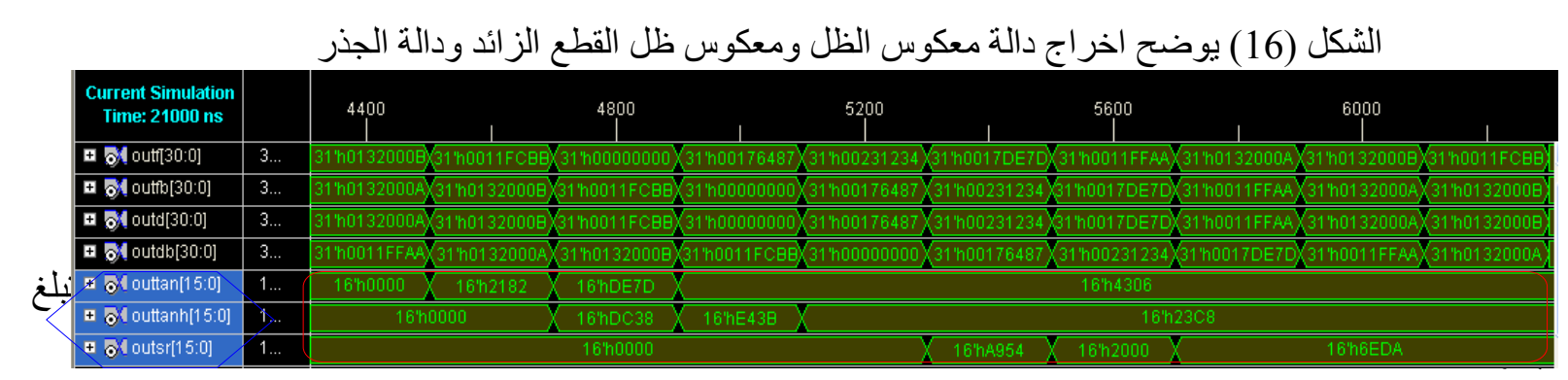




\section{3}

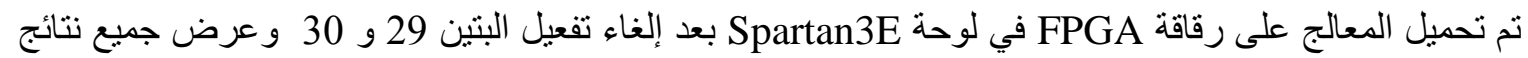

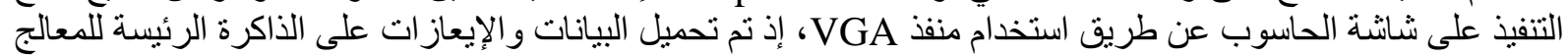

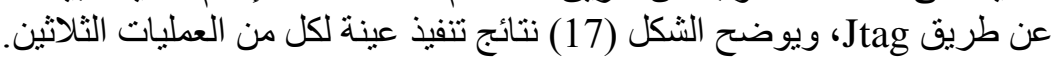

من الثكل (17) نلاحظ ان عملية محاكاة المعالج بكل عملياته مطابقة لعملية التنفيذ على شانشة الحاسوب فمثلا عملية

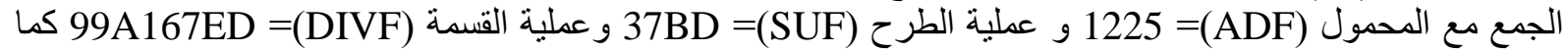
موضح في الثكل ادناه هو مطابق لنتائج الدحاكاه الموجودة في الجدول (6) و الثنكل (15) وكذللك الحال بالنسبة لباقي العمليات فكل عملية من عمليات المعالج قمنا بمحاكاتها حصلنا على نفس الناتج اثناء القيام بتنفيذ المعالج على رقاقة

.FPGA

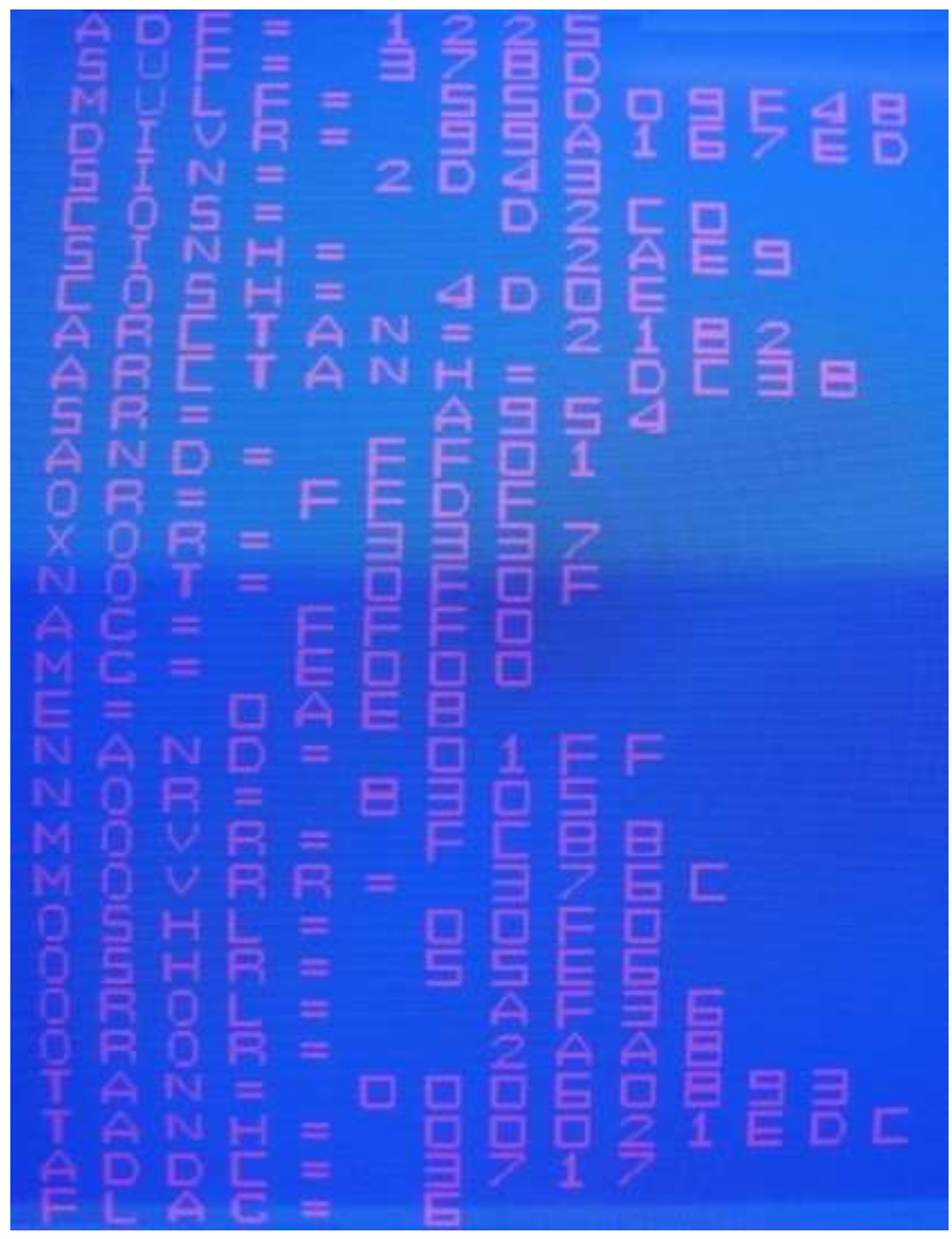

الثكل (17) نتائج تنفيذ عينة لكل من العمليات الثلاثثن على شاشة

5. الاستنتاجات : لقد نم من خلال هذا البحث استنتاج ما يأتي:-

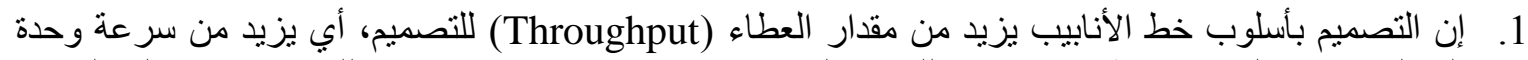

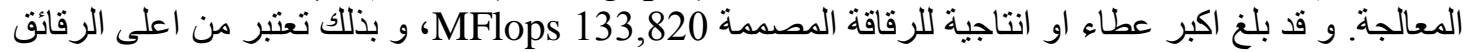

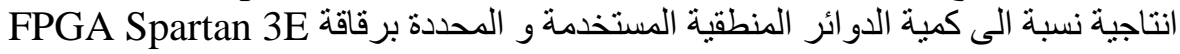




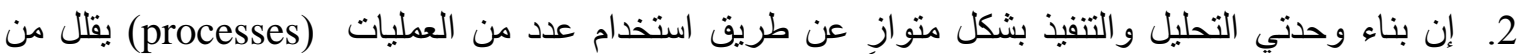

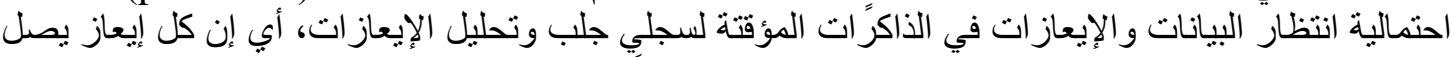

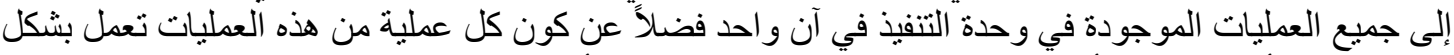

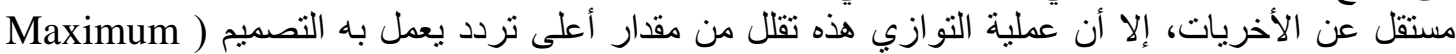
.(Operating Freq

3. عند القبام بتركيب المعالج باستخدام إحدى أدوات البرنامج ISE، لوحظ أن ذاكرتي جلب وتحليل الإيعاز ات كُوّنّت

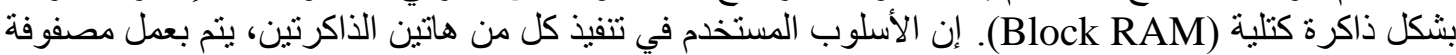

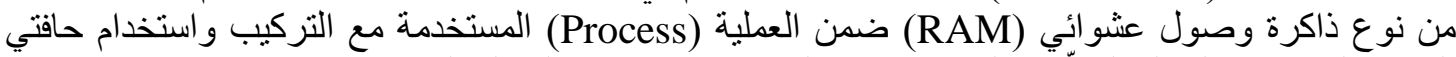

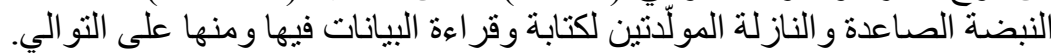

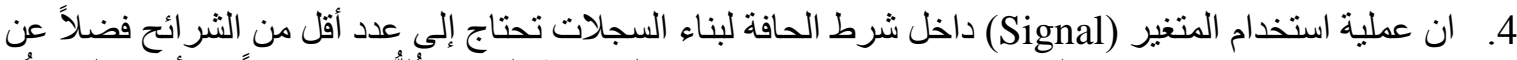

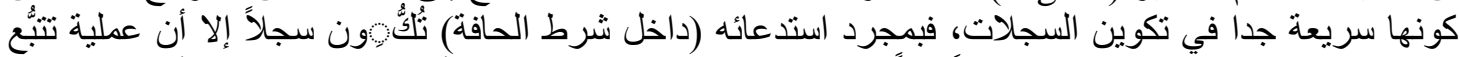

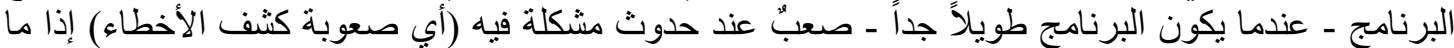

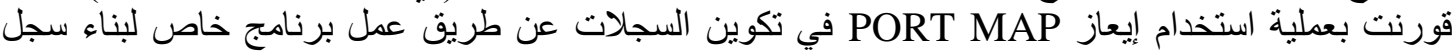

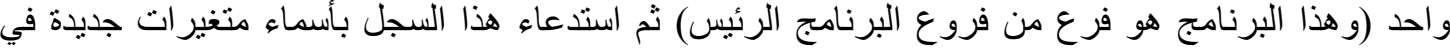
البرنامج الرئيس لتكوين سجلات بعدد الاستدعاءعات.

• عملية تحديث البيانات والإيعاز ات عن طريق عمل إعادة تهيئة جزئية أو كلبة للاكرة الرئيسة عن طريق المنفذ

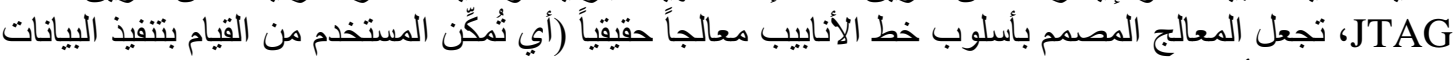

$$
\text { و الإيعازات في أي وقت وبشكل آني). }
$$

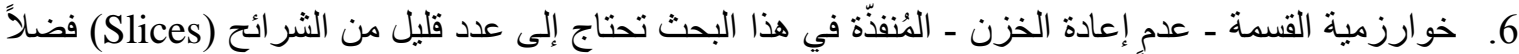

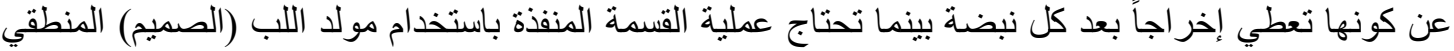
IP Core Generation يزيد من مقدار أعلى تردد تعمل به عملية القسمة.

\section{$\underline{\text { References : }}$}

[1] Joanna Armeni, "Glossary Terms Basic Concepts", http://schoolnet.gov.mt/ictsec/Resources/SharingZone/ResNo033.doc.

[2] Santa Clara, Calif.,"60 years of continued transistor shrinkage, innovation Intel 45 Nanometer Technology Gives the Transistor Dramatic Make-Over", www.intel.com/pressroom, Jan. 29, 2007.

[3] John Bruno, John W. Jones, Kimming SO,"Deterministic Scheduling with Pipelined Processors", IEEE Transactions On Computers, Vol. c-29, NO. 4, April 1980.

[4] Basil Shukr Mahmood. " Design of a pipeline Graphic Processor", Raf.jour.Sci.,Vol.7,No.1,pp.50-68, 1996.

[5] Charles Brej."A MIPS R3000 microprocessor on an FPGA", 13 February 2002. www.citeulike.org/user/rgrubisic/article/2227725.

[6] Jonathan Barre, C. Landet, Christine Rochange, Pascal Sainrat, "Modeling InstructionLevel Parallelism for WCET Evaluation", Proceedings of the 12th IEEE International Conference on Embedded and Real-Time Computing Systems and Applications (RTCSA'06), 0-7695-2676-4/06. 2006 IEEE.

[7] Ehsan Abdul-Sattar Ali, "Design and Testing A Reconfigurable Calculation Unit using FPGA" Master thesis, Computer Department, College of Engineering, Mosul University, 2008. 
[8] Jari Nurmi, "Processor Design : System-on-Chip Computing for ASICs and FPGAs", Finland Tampere University of Technology, Published by Springer, P.O. Box 17, 3300 AA Dordrecht, The Netherlands. Copyright (C 2007 Springer, www.Springer.com.

[9] Kris Gaj, "VHDL Coding for Synthesis", ECE 448 - FPGA and ASIC Design with VHDL, George Mason University, Spring 2009.

[10] "Spartan-3E FPGA Family : Complete Data Sheet", Copyright @ $2005-2008$ Xilinx, Inc. URL : www.xilinx.com/spartan3e. DS312 April 18, 2008.

[11] Petru Eles, Krzysztof Kuchcinski, Zebo Peng, Marius Minea, "Two Methods for Synthesizing VHDL Concurrent Processes", The Swedish National Board for Technical Development (NUTEK), and TEMPUS JEP-2754, Linköping University, 1993.

ftp://ftp.ida.liu.se/pub/publications/techrep/1993/r-93-22.ps.gz.

[12] Sherif Galal and Dung Pham, "Division Algorithms and Hardware Implementations", EE 213A: Advanced DSP Circuit Design, The University of California, August 2007.

ww.ee.ucla.edu/ingrid/ee213a/lectures/division_presentV2.pdf.

[13] "CORDIC v3.0," DS249 May 21, 2004 @Xilinx,Inc. http://www.xilinx.com/legal.htm.

تم اجراء البحث في كلية ألهنسسة = جامعة ألموصل 